\title{
Forces Between Aqueous Non-Uniformly Charged Colloids from Molecular Simulation
}

\author{
A. Striolo ${ }^{\mathrm{a}, \mathrm{b}}$, D. Bratko ${ }^{\mathrm{a}}$, J.Z. Wu ${ }^{\mathrm{a}, \mathrm{c}, \mathrm{d}}$, N. Elvassore ${ }^{\mathrm{b}}$, H.W. Blanch ${ }^{\mathrm{a}}$, J.M. Prausnitz ${ }^{\mathrm{a}, \mathrm{d}}$ * \\ ${ }^{a}$ Chemical Engineering Department, University of California, Berkeley, CA 94720-1462 \\ ${ }^{\mathrm{b}}$ Dipartimento di Principi e Impianti di Ingegneria Chimica, Università di Padova, Via \\ Marzolo 9, 35131 Padova, Italy \\ ${ }^{\mathrm{c}}$ Department of Chemical and Environmental Engineering, University of California, \\ Riverside, CA 92521 \\ ${ }^{\mathrm{d}}$ Chemical Sciences Division, Lawrence Berkeley National Laboratory, Berkeley, CA \\ 94720 \\ *Author to whom correspondence should be addressed
}

\begin{abstract}
NVT Monte Carlo simulation results are presented for the forces between charged colloids within the primitive model for electrolytes. The calculations show that when charged colloids have a net dipole moment, a strong attraction can arise at short separations. The attractive force is not purely electrostatic; significant contributions follow from hard-sphere collisions between the electrolyte ions and the colloidal particles. In divalent electrolyte solutions, non-uniformly charged colloids show an oscillatory force profile as a function of separation, due to layering of electrolyte ions around the interacting colloids.

Simulation results are compared to two analytical models derived from classical Debye-Hückel screened potentials. In the first model, contributions from charge-charge, dipole-dipole, and charge-dipole interactions are independently angle-averaged and then added to obtain the colloid-colloid potential. In the second model, the pair potential is obtained by simultaneously angle-averaging all interactions.

Our results show that simultaneous angle-averaging of anisotropic interactions provides significant improvement over the commonly used additivity approximation.
\end{abstract}

Key words: Monte Carlo simulation, dipolar charged colloids, potential of mean force, DLVO theory

\section{Introduction}

Interactions between electrically charged particles determine the essential properties of solutions containing latex particles, clays, or proteins [1-4]. Such colloidal solutions 
appear in many industrial processes, in particular in biotechnology, and in the food, pharmaceutical, and cosmetics industries.

Traditional theories consider the potential between charged colloids in solution as the isotropic interaction between particles characterized either by constant surface charge or by constant surface potential in a medium [1,5-8]. This approximation is satisfactory for describing highly symmetric colloids like synthetic latex or silica particles. However, many systems of industrial and biological interest, e.g. proteins, comprise charged colloidal particles with uneven charge distribution. Protein solubility can be affected by highly specific protein-protein interactions in the protein crystal [9]; because anisotropic interactions are required to fit the protein orientations in the crystal, it is likely that the protein-protein pair potential in the liquid phase is also anisotropic [4,10,11].

The effect of charge heterogeneity on colloid-colloid pair interactions has received considerable attention. For example, Yoon and Lenhoff [12] used a boundary-element method to compute the potential between a protein and a charged surface. Their results, in qualitative agreement with previous experimental reports [13], showed that ribonuclease A preferentially adsorbs onto a negatively charged surface with its active site facing the surface. Roush et al. [14] numerically solved the linearized PoissonBoltzmann equation for the electrostatic interaction between a surface and a charged sphere, observing an orientation-dependent potential energy. Grant [15] applied a boundary-element technique to study the interactions between proteins characterized by surface patchiness and charge fluctuations in electrolyte solutions. Accounting for nonuniform charge effects yielded more consistent predictions for the effective Hamaker constant obtained from experimental osmotic second virial coefficients for protein solutions. Sader and Lenhoff [16] applied the superposition approximation to study the interaction between two non-uniformly charged colloidal particles and between one nonuniformly charged particle and a surface. Their calculations reveal notable changes in the effective interaction potential due to charge heterogeneity.

Approximating protein charge distribution by a dipole may provide a reasonable representation of charged-protein electrostatic interactions in aqueous solutions $[17,18]$. McClurg and Zukoski [19] used the linearized Poisson-Boltzmann equation to estimate the electrostatic interaction energy for two charged dipolar proteins. They provided 
analytical expressions for the multipolar-expansion solution for the protein-protein interaction potential as a function of protein-protein orientation. They also provided approximate analytical expressions for such interactions where protein charge, protein size, and protein dipole moment are input parameters. They showed that electrostatic multipolar forces may be largely responsible for attractions between proteins even when they are like-charged. However, they did not provide a simple analytical expression for the angle-averaged potential of mean force between charged colloids with finite dipole moments.

Toward a better understanding of the effect of non-uniform charge distribution of charged particles on the colloid-colloid potential of mean force, we have performed simulation calculations of the orientation-averaged interaction between anisotropic charged colloids in electrolyte solutions. We study colloids with the same net charge with or without a strong permanent dipole. Colloids without dipole moment carry only a net charge in their center of mass, whereas colloids with a dipole moment carry charged groups asymmetrically distributed within the particle. We consider colloids in aqueous solutions containing mono- or divalent electrolytes at two concentrations

Limited to the conditions considered in our simulations, when charged colloids in solutions of finite-size monovalent or divalent electrolytes have no dipole, they show a repulsive force at all separations. However, when charged colloids bear a net dipole, the angle-averaged interaction can be strongly attractive. Further, when charged colloids have a dipole, the simulated colloid-colloid force profile in divalent electrolyte solution can be oscillatory due to layering of electrolyte ions around the interacting colloids

We compare our simulated potentials of mean force to results from analytical theories. To date, models based on the Derjaguin-Landau-Verwey-Overbeek (DLVO) theory [20] have allowed a qualitative, and sometimes quantitative understanding of phenomena such as the stability of charged dispersions, the short-range liquid order observed in scattering experiments [21,22], and the fluid-crystal transition at low ionic strength [23]. The DLVO expression for the screened charge-charge interaction between colloids is used in two models considered here. Following Vilker et al. [24] and Phillies [25], Prausnitz and coworkers [17] reported isotropic expressions for protein-protein interaction potentials where the protein net dipole gives dipole-dipole attraction, and where protein net charge 
and net dipole also give dipole-charge attractions. In that calculation, each contribution is computed separately by appropriate orientation averages; all the contributions are added to the DLVO term to obtain the pair potential of mean force.

Separate orientation-averaging, however, is approximately valid only in systems with relatively weak coupling between the charge-dipole and the dipole-dipole interactions. It is not valid for colloids that simultaneously carry a strong dipole and a high net charge; such colloids are considered in this work. As pointed out by Lenhoff and coworkers [11], a correct theory must be based on simultaneous orientation-averaging of all electrostatic contributions. A recent analysis along these lines by Bratko et al. [26] indeed shows that the properly angle-averaged charge-dipole and dipole-dipole interaction is less attractive than that predicted from separate averaging.

The simulations for charged dipole macroions presented here strongly support the above conclusion.

\section{Model and Simulation Details}

Canonical NVT Monte Carlo simulation was used following the algorithm proposed in our earlier works. [27,28]. Macroions and electrolytes are simulated using the primitive model for ionic solutions [29], augmented by a prescribed distribution of charged groups in the interior of the colloidal particles. The solution is described by an ensemble of hard spheres of different charge and size dispersed in a dielectric continuum whose permittivity corresponds to that of the solvent.

The average force $F$ between two aqueous colloids surrounded by small ions comprises three contributions:

$F(d)=-\left\langle\frac{\partial \varphi_{C C}(d)}{\partial d}\right\rangle-\left\langle\sum_{i=1}^{N} \frac{\partial \varphi_{i C}\left(r_{i C}\right)}{\partial d}\right\rangle+F^{H S}(d)$

Angular brackets denote ensemble averages, $d$ is the separation between two colloids, and $r_{i C}$ is the distance between a small ion $i$ and a colloid. Subscript $C$ denotes a colloid. $\varphi_{i j}$ is the pair potential between ionic particles $i$ and $j$ separated by center-to-center distance $l_{i j}$ : 
$\varphi_{i j}\left(l_{i j}\right)=\left\{\begin{array}{cc}\frac{q_{i} q_{j}}{4 \pi \varepsilon \varepsilon_{0} l_{i j}} & l_{i j} \geq\left(\sigma_{i}+\sigma_{j}\right) / 2 \\ \infty & \text { otherwise }\end{array}\right.$,

where $\varepsilon, \varepsilon_{0}, \sigma_{i}$ and $q_{i}$ are respectively relative permittivity, permittivity of vacuum $\left(\varepsilon_{0}=8.85410^{-12} \mathrm{As} / \mathrm{Vm}\right)$, diameter and charge of species $i$.

In Eq. (1), the first term on the right-hand side is the electrostatic force between the two interacting colloids. The second term accounts for electrostatic forces exerted on the colloids by the electrolyte ions. As particles cannot overlap, this term contains no contribution from hard-core interactions. The third term represents the averaged force resulting from collisions between hard-core colloids and electrolyte ions calculated by Eq.(10) of ref. [28]. All terms are computed by appropriate averages over colloid-colloid angular orientations.

According to the NVT ensemble, the number of particles in the simulation box, box volume, and temperature were kept constant during a simulation run [30]. In principle, the interaction between a pair of macroions in an electrolyte medium should be determined at constant chemical potential of small ions rather than at constant number of particles in the system [31,32]. As discussed previously [28], the error introduced by presuming a constant number of ions in the simulation system is small in comparison with the statistical uncertainty of the simulation.

A colloid with uniform charge distribution was simulated as a hard sphere that bears a net charge in its center of mass. To account for non-uniform charge distribution, a colloidal particle was charged by six positive and four negative charged groups. The charged groups were placed within the hard-sphere colloid giving rise to a permanent dipole moment. Table 1 describes the configurations of the charged groups within the colloids for the different cases considered. Fig. 1 provides a schematic representation of a non-uniformly charged macroion simulated in this work.

The conditions chosen in this work provide information about the effect of non-uniform charge distribution on the colloid-colloid pair potential of mean force between spherical particles with relatively strong dipolar interactions. Colloid charges and permanent dipoles considered in our simulations are chosen within a representative range typical for proteins in aqueous solution; for example, for $\alpha$-chymotrypsin in aqueous solution the 
dipole moment is 380D [17]). Colloid diameter was $20 \AA$. We studied solutions with monovalent or divalent electrolytes at concentrations 0.12 or $0.22 \mathrm{~mol} / \mathrm{l}$. Here electrolyte concentration refers to the added salt excluding the colloid counterions. Typical of a hydrated ion, ion diameter was set at $4 \AA$.

For the salt concentrations considered here, the simulation box size, $118 \AA$, was sufficient to render interactions between colloids in different boxes insignificant. Table 2 gives details for a typical simulation run. Periodic boundary conditions and Ewald summations [30] were used in each computer experiment.

To obtain the orientation ensemble average, in addition to the usual translational moves [28], either of the two interacting colloids was periodically randomly rotated around its center of mass. The attempted angle for each rotational move, as low as $0.02 \pi$, was such that the acceptance ratio was close to $50 \%$. The remaining details of the simulations have been described elsewhere [27,28,33]. The code was validated by reproducing previously reported simulation data by $\mathrm{Wu}$ et al. for charged colloids with zero dipole moment [28]. Four million moves were used to equilibrate the system; up to forty million movements were required in the production phase.

\section{Simulation Results}

In this section, we report simulated results for colloids in electrolyte solutions. Colloidcolloid forces and internal energies were computed at various conditions. In the next section, the simulation calculations are compared to results from analytical models.

Fig. 2 shows colloid-colloid forces for $+2 \mathrm{e}_{0}$ colloids with either zero or $95 \mathrm{D}$ dipole in 0.12M monovalent electrolyte solution (cases A and B in Table 1). Fig. 2A is for the total colloid-colloid force, Eq.(1), while Figs. $2 \mathrm{~B}$ and 2C are for the electrostatic (first two terms on the right-hand side of Eq.(1)) and the hard-sphere collision force (last term in Eq.(1)) respectively. In both cases, the total force is repulsive at all separations. As expected, at short colloid-colloid separations the repulsion is weaker for dipolar (case-B) colloids. The weaker repulsion is due both to the electrostatic and to the hard-sphere collision components of the colloid-colloid force. Comparing the components of the colloid-colloid force, the hard-sphere collision force (Fig. 2C) appears similar in the two cases. At very short separations, the hard-sphere collision force is slightly more attractive 
for dipolar colloids, indicating a depletion of small ions in the region between the interacting charged dipolar colloids. However, the reduction in repulsion arises primarily from correlated dipole-dipole interactions, because the electrostatic component (Fig. 2B) is less repulsive at small colloid-colloid separations for dipolar colloids.

Fig. 3 shows the total internal energy for the interacting pair of colloids considered in Fig. 2. The internal energy is strongly repulsive near contact and weakly attractive at bigger separations. Previously reported results [27] showed that the internal energy for $+20 \mathrm{e}_{0}$ apolar colloids in $0.5 \mathrm{M}$ monovalent electrolyte solutions was repulsive at short colloid-colloid separations, whereas it was attractive at intermediate separations. A rather shallow energy minimum is revealed in the present calculation because the charge density we consider is significantly smaller. At decreasing colloid-colloid separations we show the internal energy to increase faster for colloids without dipoles because of favorable colloid-colloid orientations available to dipolar colloids

Fig. 4 shows colloid-colloid forces computed for macroions with net charge $+8 \mathrm{e}_{0}$ in 0.12M monovalent electrolyte solution. Different symbols represent results obtained for colloids with different charge distributions. Colloids considered correspond to cases C, D, and $\mathrm{E}$ shown in Table 1. Their finite dipoles are, respectively, zero, 380, and 490D. While the total force (Fig. 4A) is repulsive at all colloid-colloid separations when the macroions have no dipole, it is attractive, at low separations, when macroions have a finite dipole moment. For distances larger than 1.5 times the colloid diameter, the colloidcolloid force appears slightly repulsive for all cases considered here. The total force is dominated by electrostatic interactions. As shown in Fig. 4B, the general features of the electrostatic forces resemble those observed for the total force (Fig. 4A). At intermediate separations, the electrostatic interactions between colloidal particles with 380D dipole is more repulsive than that for charged particles without dipoles. This counter-intuitive result can be attributed to the depletion of small ions between the interacting colloids as indicated in the force profiles due to the hard-sphere-collision term (Fig. 4C). The depletion of small ions weakens the screening of charge-charge repulsion. For 490D colloids, the electrostatic interaction is dominated by charge-dipole and dipole-dipole attractions, except for distances exceeding $\sim 1.4 \sigma_{C}$. The hard-sphere collision component (Fig. 4C), repulsive at all separations in the absence of dipoles, is attractive when the 
interacting colloids have net dipoles, a consequence of the depletion of small ions between macroions as they tend to orient in a way minimizing the distances between oppositely charged groups on adjacent macro-particles. As discussed in our earlier work [33], we generally observe a depletion of simple ions in the region between oppositely charged macroion domains.

Fig. 5 shows the total internal energy for the interacting pairs of colloids for the cases considered in Fig. 4. While the internal energy of dipole-free colloids increases with decreasing colloid-colloid separation, it decreases when the colloids have dipoles because of attractive energy associated with favorable colloid-colloid orientations. Because of the high dipole moments in these calculations, attractive dipolar interactions outweigh charge-charge repulsion at all separations. Previously reported results [27] showed that the internal energy for $+20 \mathrm{e}_{0}$ apolar colloids in $0.5 \mathrm{M}$ monovalent electrolyte solutions is repulsive at short colloid-colloid separations, and attractive at intermediate separations. At the present conditions, the observed long-ranged attraction is weak because the charges on the colloids are significantly smaller.

Figs. 6, 7 and 8 compare force profiles for pairs of $+8 \mathrm{e}_{0}$ colloids without (Fig. 6) or with (Figs. 7 and 8) dipole moments in solutions of various ionic strengths. As expected, increasing the ionic strength reduces electrostatic repulsion between charged apolar colloids (Fig. 6B. When $+8 \mathrm{e}_{0}$ apolar colloids are interacting in divalent electrolyte solutions of concentrations 0.12 or $0.22 \mathrm{M}$, the colloid-colloid repulsion is weaker than that computed in monovalent electrolyte solutions of the same concentrations because stronger screening is provided by divalent ions. At the electrolyte concentrations here considered, simulation results do not reveal any significant influence of divalent electrolyte concentration on the colloid-colloid force. This result follows from excludedvolume effects: finite-size ions are excluded from the region between the interacting colloids. The electrostatic component of the force (Fig. 6B) shows a weak colloid-colloid attraction at distances around $\sim 1.3 \sigma_{C}$. in divalent electrolyte solutions, as noted in previous simulations [27,28,34,35]. However, the hard-sphere collision repulsion outweighs the electrostatic attraction, and the total force is essentially repulsive, within statistical uncertainty, at all separations. Evidently, the charge density considered in the present simulations is too low to give rise to net attraction due to ion-ion correlation 
effects. In our simulations, the hard-sphere-collision component of the colloid-colloid force does not show any significant dependence upon solution ionic strength (Fig. 6C).

For $+8 \mathrm{e}_{0}, 380 \mathrm{D}$ colloids (case D, Fig. 7), increasing monovalent electrolyte concentration appears to weaken colloid-colloid repulsion at distances beyond $\sim 1.5$ $\sigma_{C}$.(Fig. 7A). Within computational accuracy, the electrostatic component of the colloidcolloid force (Fig. 7B) does not depend visibly upon the bulk electrolyte concentration at reduced distances lower than 1.4. This result is probably related to the depletion of small ions between favorably oriented macroions [36]. Attractive collision force at small separations (Fig. 7C) confirms that the ion concentration between colloids facing each other's oppositely charged domain remains relatively low. Hence, small ions do not screen the electrostatic colloid-colloid repulsion as much as the DLVO theory predicts. At reduced distances between 1.5 and 1.7 the hard-sphere collision force is more attractive at lower electrolyte concentrations. These results, clearly associated with excluded-volume effects of layering of simple ions, cannot be interpreted within the framework of DLVO and related theories. Integral-equation theory, augmented by dipolerelated interactions, is required to capture the segregation effect of simple ions between adjacent macro-particles (see, for example, ref. [36]).

Fig. 8 shows the force profile between $+8 \mathrm{e}_{0}, 380 \mathrm{D}$ colloids (case-E in Table 1) in $0.12 \mathrm{M}$ divalent electrolyte solutions. Because divalent electrolytes more effectively screen the electrostatic repulsion, the colloid-colloid force is more attractive than that simulated in monovalent electrolyte solutions. The force is strongly oscillating, even though the interaction is predominantly attractive. An oscillatory force was measured at close separations between mica surfaces in various solvents due to structuring of solvent layers [37-40]. Analogous oscillations observed in the present study follow from layering of electrolyte ions packed between the colloid spheres, further enhanced by strong ionion correlations in divalent electrolytes [41]. At higher divalent electrolyte concentration $(0.22 \mathrm{M})$, the packing effects and concomitant force oscillations become more pronounced. Repulsive peaks in the force profile increase and the attractive well, significant at reduced colloid-colloid distance 1.3, becomes deeper at higher electrolyte concentration. In view of longer equilibration times required at higher electrolyte 
concentrations, only some representative results are given for colloids in $0.22 \mathrm{M}$ divalent electrolyte solutions.

The repulsive peak position $\left(1.2 \sigma_{C}\right)$ corresponds to a separation sufficient to accommodate one electrolyte ion between the two interacting colloids. At reduced distances above 1.2, the sign of the hard-sphere-collision force (Fig. 8C) is opposite to that of the electrostatic force (Fig. 8B). At reduced colloid-colloid distances below 1.2, the hard-sphere-collision force is attractive because of the osmotic effect, and because of the attractive electrostatic force between favorably oriented dipolar macroions. The two contributions result in strong colloid-colloid attraction at short separations.

Fig. 9 shows the total internal energy for interacting pairs of $+8 \mathrm{e}_{0}$ colloids in solutions of various ionic strengths. Figs. 9A and 9B are, respectively, for colloids with no dipole and colloids with 380D dipole (case-C and case-D colloids, see Table 1). At increasing monovalent electrolyte concentration, the internal energy depends only weakly on the salt concentration for charged colloids with no dipole moment. For charged apolar colloids in divalent electrolyte solutions, the internal energy is weakly attractive even at near-contact separations, whereas it is repulsive in monovalent electrolyte solution (Fig. 9A). For charged apolar colloids in $0.22 \mathrm{M}$ divalent electrolyte solution, the internal energy is weakly repulsive at intermediate separations, but remains attractive at short distances. However, results in divalent electrolyte solutions are affected by significant statistical uncertainty.

For dipolar colloids in monovalent electrolyte solutions, the internal energy profile is independent of electrolyte concentration for reduced distances above 1.6, but at shorter distances the internal energy becomes somewhat less attractive with increasing electrolyte concentration, probably because of enhanced screening of the multi-polar attraction.

In divalent electrolyte solutions, the internal energy for pairs of charged dipolar colloids (case D in Table 1) displays stronger oscillations indicating pronounced packing of finitesize ions around the interacting colloids. However, the internal energy is strongly attractive at contact and at reduced colloid-colloid separations of about 1.2. 


\section{Comparison with Theory}

As discussed in earlier works $[17,18,24,25,42]$, interactions between dipolar colloids or proteins can be approximately described in terms of electrostatically screened chargecharge $(q-q)$, angle-averaged charge-dipole $(q-\mu)$, and angle-averaged dipole-dipole $(\mu-\mu)$ interactions. Typically, Boltzmann averages of each type of colloid-colloid interactions over the colloid-colloid orientations are first calculated separately [18,42]. Then, the different angle-averaged contributions are summed to obtain the colloid-colloid potential of mean force, $W(d)$, as a function of the colloid-colloid separation $d$ :

$W(d)=W_{q-q}(d)+W_{q-\mu}(d)+W_{\mu-\mu}(d)$.

In Eq.(3), $W_{q-q}$ is the screened charge-charge repulsion, $W_{q-\mu}$ is the screened charge-dipole attraction, $W_{\mu-\mu}$ is the screened dipole-dipole attraction. Within the Debye-Hückel approximation for charge and dipole screening effects [25], the individual terms are given by $[17,25]$ :

$$
\begin{aligned}
& \beta W_{q-q}(d)=\frac{\beta q_{i} q_{j}}{4 \pi \varepsilon \varepsilon_{0} d} S_{q-q}(d, \kappa), \\
& \beta W_{q-\mu}(d)=-\frac{1}{3} \frac{\beta^{2} q^{2} \mu^{2}}{\left(4 \pi \varepsilon \varepsilon_{0}\right)^{2} d^{4}} S_{q-\mu}(d, \kappa), \\
& \beta W_{\mu-\mu}(d)=-\frac{9}{2} \frac{\beta^{2} \mu^{4}}{\left(4 \pi \varepsilon \varepsilon_{0}\right)^{2} d^{6}} S_{\mu-\mu}(d, \kappa) .
\end{aligned}
$$

In Eqs. (4-5), $S_{i}$ are screening factors given by:

$$
\begin{aligned}
& S_{q-q}(d, \kappa)=\frac{\exp \left[-\kappa\left(d-\sigma_{C}\right)\right]}{\left[1+\left(\frac{\kappa \sigma_{C}}{2}\right)\right]^{2}}, \\
& S_{q-\mu}(d, \kappa)=\left\{\frac{3(1+\kappa d) \exp \left[-\kappa\left(d-\sigma_{C}\right)\right]}{\left(1+\left(\frac{\kappa \sigma_{C}}{2}\right)\left[2+\kappa \sigma_{C}+\left(\frac{\kappa \sigma_{C}}{2}\right)^{2}+\left(1+\left(\frac{\kappa \sigma_{C}}{2}\right)\right) \frac{\varepsilon_{S}}{\varepsilon}\right]\right.}\right\}^{2},
\end{aligned}
$$




$$
S_{\mu-\mu}(d, \kappa)=\frac{\left(2+2 \kappa d+(\kappa d)^{2}\right)^{2}+2(1+\kappa d)^{2} \mid \exp \left[-2 \kappa\left(d-\sigma_{C}\right)\right]}{\left[2+\kappa \sigma_{C}+\left(\frac{\kappa \sigma_{C}}{2}\right)^{2}+\left(1+\left(\frac{\kappa \sigma_{C}}{2}\right)\right) \frac{\varepsilon_{S}}{\varepsilon}\right]^{4}}
$$

Here $\varepsilon_{S}$ is the permittivity of the colloid particle, assumed to be equal to that of the electrolyte medium.

As pointed out by Lenhoff and coworkers [11], adding the distinct orientation-averaged terms could introduce deviations from the correct effective pair potential due to neglecting correlations between charge-dipole and dipole-dipole terms when both of the interacting particles carry a net charge together with a dipole. In this case, opposite colloid-colloid orientations are favored by charge-dipole and dipole-dipole interactions. Angle-averaged charge-dipole and dipole-dipole terms are therefore strongly nonadditive. Recently, we [26] proposed an approximate analytic expression for the potential of mean force between colloids characterized simultaneously by finite charge and finite dipole (see Fig. 1). We noted [26] that the potential of mean force between non-uniformly charged colloids, when dipole-related interactions are comparable to or exceed the thermal energy, cannot be computed by assuming a pair-wise sum of distinct orientation averages for charge-dipole and dipole-dipole interactions. Using a simple discreteorientation model, we obtained an approximate expression for the potential of mean force which exactly reproduces the correct behavior in weak and strong coupling limits, and appears appropriate for practical calculations. In this approximation, the orientation space of the two dipoles is discretized, with each dipole sampling only six principal directions. The model is applied to estimate the coupling correction, i.e. the difference between the total potential of mean force and a pairwise sum of uncoupled charge-dipole and dipoledipole contributions. Adding the perturbation term to the sum of distinct contributions from the continuous representation $[25,42]$ gives[26]:

$$
\begin{aligned}
& \beta W(d)=\beta W_{q-q}(d)- \\
& \ln \frac{\left(4+4 \cosh \alpha_{3}+\exp \left(-2 \alpha_{2}\right)+8 \cosh \alpha_{1}+\exp \left(2 \alpha_{2}\right) \cosh 2 \alpha_{1}\right)\left(\frac{\sinh \alpha_{1}}{\alpha_{1}}\right)^{2}}{2\left(2+\cosh \alpha_{1}\right)^{2}} .
\end{aligned}
$$


In Eq. (10), $W_{q-q}$ is given in Eq. (4). The other terms are:

$$
\begin{aligned}
& \alpha_{1}=\frac{\beta q \mu}{4 \pi \varepsilon \varepsilon_{0} d^{2}} S_{1}(d, \kappa), \\
& \alpha_{2}=-\frac{\beta \mu^{2}}{4 \pi \varepsilon \varepsilon_{0} d^{3}} S_{2}(d, \kappa), \\
& \alpha_{3}=-\frac{\beta \mu^{2}}{4 \pi \varepsilon \varepsilon_{0} d^{3}} S_{3}(d, \kappa) .
\end{aligned}
$$

In Eqs. (11-13), $S_{i}$ are screening factors obtained by:

$$
\begin{aligned}
& S_{1}(d, \kappa)=\frac{3 \exp \left[-\kappa\left(d-\sigma_{C}\right)\right](1+\kappa d)}{\left[1+\frac{\kappa \sigma_{C}}{2}\right]\left[2+\kappa \sigma_{C}+\frac{\left(\kappa \sigma_{C}\right)^{2}}{4}+\left(1+\frac{\kappa \sigma_{C}}{2}\right) \frac{\varepsilon_{S}}{\varepsilon}\right]}, \\
& S_{2}(d, \kappa)=\frac{9 \exp \left[-\kappa\left(d-\sigma_{C}\right)\right]\left(1+\kappa d+\frac{(\kappa d)^{2}}{2}\right)}{\left[2+\kappa \sigma_{C}+\frac{\left(\kappa \sigma_{C}\right)^{2}}{4}+\left(1+\frac{\kappa \sigma_{C}}{2}\right) \frac{\varepsilon_{S}}{\varepsilon}\right]^{2}}, \\
& S_{3}(d, \kappa)=\frac{9 \exp \left[-\kappa\left(d-\sigma_{C}\right)(1+\kappa d)\right.}{\left[2+\kappa \sigma_{C}+\frac{\left(\kappa \sigma_{C}\right)^{2}}{4}+\left(1+\frac{\kappa \sigma_{C}}{2}\right) \frac{\varepsilon_{S}}{\varepsilon}\right]^{2}} .
\end{aligned}
$$

Below, we compare predictions of the two analytical models, [Eq.(3) and Eq.(10)], with our simulation results. In view of the shortcomings of DLVO-based models where simple ions are point charges and ion-ion correlations are ignored, these models cannot reproduce the details of colloid-colloid interactions at small separations. The deviations of both Eq.(3) and Eq.(10) from simulation results are further exacerbated in the presence of dipoles because the theory neglects coupling between charge and dipole screening effects.

When the colloids carry a net charge but have no dipole, the two analytical models reduce to the DLVO approximation expressed by Eq.(4) [20]. Fig. 10 compares potentials of mean force from computer simulations with predictions of DLVO theory for zero polar $+2 \mathrm{e}_{0}$ and $+8 \mathrm{e}_{0}$ colloids in $0.12 \mathrm{M}$ monovalent electrolyte solutions. The theory reproduces the colloid-colloid potential of mean force for colloids with small charge (case A colloids), while it slightly over-estimates the repulsive potential of mean force for $+8 \mathrm{e}_{0}$ 
colloids. Such discrepancies are not surprising as DLVO theory cannot always accurately describe short-distance behavior affected by ion-ion correlations [43]. Further, the finitesize counter-ions can form densely packed micro-structured clouds between interacting colloids that may generate short-separation repulsive colloid-colloid interaction [37,38].

Fig. 11 compares the simulated colloid-colloid potential of mean force for $+8 \mathrm{e}_{0}, 380 \mathrm{D}$ and 490D colloids (cases D and $\mathrm{E}$ in Table 1) in $0.12 \mathrm{M}$ monovalent electrolyte solutions, with results obtained by both models considered here [Eq.(3) and Eq.(10)]. Neither model captures the strong colloid-colloid repulsion at intermediate separations because the analytic approximations used here are asymptotic expressions, valid at large separations, and ignore finite ion size, important at short colloid-colloid separations. Further, ion-ion correlations are not considered. For small separations, Eq.(3) gives a strong colloidcolloid attraction that does not agree with simulation. Eq.(10), on the other hand, gives a colloid-colloid potential of mean force qualitatively similar to simulation results. The attractive part of the theoretical potential closely resembles simulation results for the 490D case (case-E colloids in Table 1).

Eq.(3) overestimates the colloid-colloid attraction because it assumes additivity of several separately angle-averaged electrostatic contributions that should not be treated as uncorrelated events. These results reaffirm our previous conclusion [26] that the additivity approximation cannot be applied to charged colloidal systems in electrolyte solutions when the colloidal particles simultaneously carry strong dipoles and strong charges.

Fig. 12 shows simulated and predicted potentials of mean force for $+8 \mathrm{e}_{0}$, zero-dipole and 380D colloids (case C and D in Table 1) in $0.12 \mathrm{M}$ divalent electrolyte solution. For apolar colloids, the theory strongly overestimates the repulsion between like charged estimates of screening effects lead to serious errors in multivalent electrolyte solutions. As a result, neither model captures the repulsive potential of mean force between dipolar colloids at intermediate separations. However, while Eq.(3) predicts colloid-colloid attraction at distances shorter than $1.5 \sigma_{C}$, Eq.(10) reproduces the colloids at small separations, probably because the shortcomings of Debye-Hückel qualitative features of the effective colloid-colloid potential of mean force, viz. a weak repulsion at reduced distances above 1.3, and an attraction at shorter separations. While approximate, Eq.(10) 
represents the properties of charged colloidal suspensions much better than Eq.(3), also in divalent electrolyte solutions.

\section{Conclusions}

We report Monte Carlo simulations of electrostatic forces between charged dipolar colloid particles in electrolyte solutions containing ions of finite size. Colloids considered carry a net charge of either +2 or $+8 \mathrm{e}_{0}$; they are characterized either by a zero or a finite dipole moment. The dipole moment is generated by displacing point charges within the colloid hard sphere. Colloid-colloid forces are computed in monovalent or divalent electrolyte solutions of various concentrations.

For conditions considered in this work, while in monovalent electrolyte solutions the colloid-colloid force is repulsive when the colloids have no dipole, it can be strongly attractive when dipoles are present. The attraction has two causes: the direct chargedipole and dipole-dipole interaction, and depletion of electrolyte ions between favorably oriented dipolar colloidal particles.

The colloid-colloid force computed in divalent electrolyte solution shows significant oscillations due to layering of electrolyte ions around the colloid molecules. Monovalent electrolyte ions are not sufficiently attracted to the colloids to show similar strong coordination around the interacting macroions.

Potentials of mean force obtained from simulations are compared to results obtained by DLVO-related models. The screened charge-charge repulsive interaction is obtained from the original DLVO theory. Because of its inherent approximations, the DLVO theory provides only asymptotic expressions valid at large colloid-colloid separations. At the conditions of our present work, it provided a satisfactory description of the interaction between apolar colloids surrounded by monovalent salt but overestimates the colloidcolloid repulsion in the presence of divalent ions.

In the additivity approximation, various colloid-colloid interactions (dipole-dipole and charge-dipole) are computed independently by appropriate Boltzmann-weighted angle averages and then summed to obtain the potential of mean force between colloids in electrolyte solutions. However, Bratko et al. [26] simultaneously angle-averaged dipole- 
dipole, and charge-dipole contributions to the potential of mean force. The effective colloid-colloid potential is obtained by suitable averages over representative relative colloid-colloid orientations, leading to significant improvement over results obtained from the additivity approximation.

\section{Acknowledgments}

For helpful discussions and encouragement, A.S. thanks R. Curtis and R. Wimberly. Simulation calculations were performed on T3E machines at the computing centers of Berkeley, California, USA (NERSC), San Diego, California, USA (NPACI), Bologna, Italy (CINECA). A.S. and N.E. are grateful to the University of Padova for a 'Progetto di Ricerca per Giovani Ricercatori' fellowship. D.B., H.W.B., and J.M.P. acknowledge support from the National Science Foundation and from the Office for Basic Energy Sciences of the US Dept. of Energy. 


\section{References}

1. L. Belloni J. Phys.: Condens. Matter 12 (2000) R549-R587.

2. J. Israelachvili, Intermolecular and Surface Forces, Academic Press, London, 1991.

3. J.M. Prausnitz, Lichtenthaler, E.G. de Azevedo, Molecular Thermodynamics of Fluid-Phase Equilibria, $3^{\text {rd }}$ Ed., Prentice Hall, 1999.

4. D.F. Rosenbaum, A. Kulkarni, S. Ramakrishnan, C.F. Zukoski J. Chem. Phys. 111 (1999) 9882.

5. J.P. Hansen, H. Lowen Annual Rev. of Phys. Chem. 51 (2000) 209.

6. S.L. Carnie, D.Y.C. Chan J. Coll. Int. Sci. 155 (1993) 297.

7. J.E. Sanchez-Sanchez, M. Lozada-Casson Chem. Phys. Lett. 190 (1992) 202.

8. S.A. Palkar, A.M. Lenhoff J. Coll. Int. Sci. 165 (1994) 177.

9. M.L. Grant, D.A. Saville J. Coll. Int. Sci. 171 (1995) 35.

10. A.M. Kierzek, P. Zielenkiewicz Biophysical Chem. 91 (2001) 1-20.

11. B.L. Neal, D. Asthagiri, O.D. Velev, A.M. Lenhoff, E.W. Kaler, J. Crystal Growth 196 (1999) 377-387.

12. B.J. Yoon, A.M. Lenhoff J. Phys. Chem. 96 (1992) 3130.

13. C.S. Lee, G. Belfort Proc. Natl. Acad. Sci. U.S.A. 86 (1989) 8392-8396.

14. D.J. Roush, D.S. Gill. R.C. Willson Biphys. J. 66 (1994) 1290.

15. M.L. Grant J. Phys. Chem. B 105 (2001) 2858.

16. J.F. Sader, A.M. Lenhoff J. Coll. Int. Sci. 201 (1998) 233.

17. C.J. Coen, H.W. Blanch, J.M. Prausnitz, AIChE J. 41 (1995) 996.

18. C.J. Coen, J. Newman, H.W. Blanch, J.M. Prausnitz J. Coll. Int. Sci. 177 (1996) 276.

19. R.B. McClurg, C.F. Zukoski J. Coll. Int. Sci. 208 (1998) 529.

20. E.J.W. Verwey, J.Th.G. Overbeek, Theory of Stability of Lyophobic Colloids, 1948, Amsterdam, Elsevier.

21. S.H. Chen Ann. Rev. Phys. Chem. 37 (1986) 351.

22. C. Bonnet-Gonnet, L. Belloni, B. Cabane Langmuir 10 (1994) 4012.

23. M. Robbins, K. Kremer, G.S. Grest J. Chem. Phys. 88 (1988) 3286.

24. V.L. Vilker, C.K. Colton, K.A. Smith J. Coll. Int. Sci. 79 (1981) 548.

25. G.D.J. Phillies J. Chem. Phys. 60 (1974) 2721. 
26. D. Bratko, A. Striolo, J. Wu, H.W. Blanch, J.M. Prausnitz, J. Phys. Chem. B. (2002), in press..

27. J.Z. Wu, D. Bratko, J.M. Prausnitz, Proc. Natl. Acad. Sci. USA 95, 15169, 1998.

28. J.Z. Wu, D. Bratko, H. W. Blanch, J.M. Prausnitz, J. Chem. Phys. 111, 1999, 7084; ibid, 113, 3360 (2000).

29. H.L. Friedman, Ionic Solution Theory, Wiley, New York, 1962.

30. M.P. Allen, D.J. Tildesley, Computer Simulation of Liquids, Claredon Press, Oxford, 1987.

31. P. Attard J. Chem. Phys. 91 (1989) 3083.

32. G. Karlstrom Chem. Scr. 25 (1985) 89.

33. J.Z. Wu, D. Bratko, H.W. Blanch, J.M. Prausnitz Phys. Rev. E 62 (2000) 5273.

34. V. Lobaskin, P. Linse J. Chem. Phys. 109 (1998) 3530.

35. B. Hribar, V. Vlachy J. Phys. Chem. B 101 (1997) 3457.

36. P. Attard, S.J. Miklavic J. Chem. Phys. 99 (1993) 6078.

37. R.G. Horn, J.N. Israelachvili Chem. Phys. Lett. 71 (1980) 192.

38. R.M. Pashley, J.N. Israelachvili J. Coll. Int. Sci. 101 (1984) 511.

39. R. Kjellander, S. Marcelja, R.M. Pashley, J.P. Quirk J. Phys. Chem. 92 (1988) 6489.

40. R. Kjellander, S. Marcelja, R.M. Pashley, J.P. Quirk J. Chem. Phys. 92 (1990) 4399.

41. R. Kjellander, S. Marcelja Chem. Phys. Lett. 112 (1984) 49.

42. S.J. Miklavcic Phys. Rev. E 56 (1997) 1142.

43. R. Kjellander, S. Marcelja Chem. Phys. Lett. 127 (1986) 402. 
Table 1: Charge position within the colloids for each case studied by simulation.

\begin{tabular}{|c|c|c|c|c|}
\hline & $x / \AA$ & $y / \AA$ & $z / \AA$ & $q / e_{0}$ \\
\hline case $\mathbf{A}$ & 0.00 & 0.00 & 0.00 & +2 \\
\hline \multicolumn{5}{|l|}{$\mu=0 \mathrm{D}$} \\
\hline \multicolumn{5}{|l|}{$\mathrm{Q}=+2 \mathrm{e}$} \\
\hline case B & 0.00 & 0.00 & 7.00 & -1 \\
\hline$\mu=95 \mathrm{D}$ & 0.00 & 0.00 & -7.00 & -1 \\
\hline \multirow[t]{8}{*}{$\mathrm{Q}=+2 \mathrm{e}$} & 0.00 & 4.95 & 4.95 & -1 \\
\hline & 0.00 & -4.95 & 4.95 & -1 \\
\hline & 4.95 & 0.00 & 4.95 & +1 \\
\hline & -4.95 & 0.00 & 4.95 & +1 \\
\hline & 4.95 & 0.00 & -4.95 & +1 \\
\hline & -4.95 & 0.00 & -4.95 & +1 \\
\hline & 0.00 & 4.95 & -4.95 & +1 \\
\hline & 0.00 & -4.95 & -4.95 & +1 \\
\hline case $\mathrm{C}$ & 0.00 & 0.00 & 0.00 & +8 \\
\hline \multicolumn{5}{|l|}{$\mu=0 \mathrm{D}$} \\
\hline \multicolumn{5}{|l|}{$\mathrm{Q}=+8 \mathrm{e}$} \\
\hline case D & 0.00 & 0.00 & 7.00 & -4 \\
\hline$\mu=380 D$ & 0.00 & 0.00 & -7.00 & -4 \\
\hline \multirow[t]{8}{*}{$\mathrm{Q}=+8 \mathrm{e}$} & 0.00 & 4.95 & 4.95 & -4 \\
\hline & 0.00 & -4.95 & 4.95 & -4 \\
\hline & 4.95 & 0.00 & 4.95 & +4 \\
\hline & -4.95 & 0.00 & 4.95 & +4 \\
\hline & 4.95 & 0.00 & -4.95 & +4 \\
\hline & -4.95 & 0.00 & -4.95 & +4 \\
\hline & 0.00 & 4.95 & -4.95 & +4 \\
\hline & 0.00 & -4.95 & -4.95 & +4 \\
\hline case $\mathbf{E}$ & 0.00 & 0.00 & 9.00 & -4 \\
\hline$\mu=490 D$ & 0.00 & 0.00 & -9.00 & -4 \\
\hline \multirow[t]{8}{*}{$\mathrm{Q}=+8 \mathrm{e}$} & 0.00 & 6.36 & 6.36 & -4 \\
\hline & 0.00 & -6.36 & 6.36 & -4 \\
\hline & 6.36 & 0.00 & 6.36 & +4 \\
\hline & -6.36 & 0.00 & 6.36 & +4 \\
\hline & 6.36 & 0.00 & -6.36 & +4 \\
\hline & -6.36 & 0.00 & -6.36 & +4 \\
\hline & 0.00 & 6.36 & -6.36 & +4 \\
\hline & 0.00 & -6.36 & -6.36 & +4 \\
\hline
\end{tabular}

$\mu$ is the finite dipole moment of the macroion.

$\mathrm{Q}$ is the finite charge of the macroion.

$\mathrm{x}, \mathrm{y}$, and $\mathrm{z}$ are the coordinates, relative to the center of mass, of each charged group displaced within the macroion.

$\mathrm{q}$ is the finite charge of the each charged group within the macroion. 
Table 2: Details for a typical simulation run to determine the force between two charged, dipolar particles.

\begin{tabular}{ll}
\hline Box size & $118 \AA$ \\
Colloid diameter & $20 \AA$ \\
Colloid net charge & $+8 \mathrm{e}_{0}$ \\
No. of counter ions & 141 \\
Counter-ion charge & $-1 \mathrm{e}_{0}$ \\
Counter-ion diameter & $4 \AA$ \\
No. of co ions & 125 \\
Co-ion charge & $+1 \mathrm{e}_{0}$ \\
Co-ion diameter & $4 \AA$ \\
Ionic strength & $0.12 \mathrm{M}$ \\
Temperature & $298 \mathrm{~K}$ \\
Dielectric constant & 78.54 \\
Bjerrum length & $7.13 \AA$ \\
\hline
\end{tabular}




\section{Figure captions}

Figure 1. Schematic representation of a charged dipolar colloid simulated in this work. On the left side, a simulated colloid is represented as a hard sphere with the system of Cartesian coordinates centered in its center of mass. Six positive (black dots) and four negative (white dots) charged groups are placed within the colloidal particle according to details given in Table 1. On the right side the macroion is represented as a hard sphere with a finite charge and a finite dipole moment in its center. The left-side representation corresponds to the macroion as simulated in the calculations presented here, while the right-side representation is used in analytical models.

Figure 2. Simulated colloid-colloid reduced force as a function of reduced colloid-colloid separation for colloids with net charge $+2 \mathrm{e}_{0}$ in $0.12 \mathrm{M}$ monovalent electrolyte solution. $l_{B}$ is Bjerrum length, and $\sigma_{C}$ is colloid diameter. Fig. 2A shows the total colloid-colloid force $\left(\mathrm{F}_{\mathrm{tot}}\right)$; $2 \mathrm{~B}$ shows the electrostatic component of the total force $\left(\mathrm{F}_{\mathrm{el}}\right)$, and Fig. $2 \mathrm{C}$ shows the hard-sphere-collision component $\left(\mathrm{F}_{\mathrm{HS}}\right)$. Full diamonds are for zero dipolar colloids (case A in Table 1); open diamonds are for dipolar colloids (case B). Lines are guides to the eye.

Figure 3. Reduced total internal energy for a pair of interacting colloids as a function of reduced colloid-colloid separation. $\sigma_{C}$ is colloid diameter. Full diamonds are for $+2 \mathrm{e}_{0}$, zero dipolar colloids (case A in Table 1); empty diamonds are for 95D colloids (case B). Lines are guides to the eye.

Figure 4. Simulated colloid-colloid reduced force as a function of reduced colloid-colloid separation for colloids with net charge $+8 \mathrm{e}_{0}$ in $0.12 \mathrm{M}$ monovalent electrolyte solution. $l_{B}$ is Bjerrum length, and $\sigma_{C}$ is colloid diameter. Fig. 4A shows the total colloid-colloid force $\left(\mathrm{F}_{\text {tot }}\right)$; $4 \mathrm{~B}$ shows the electrostatic component of the total force $\left(\mathrm{F}_{\mathrm{el}}\right)$, and $4 \mathrm{C}$ shows the hard-sphere-collision component $\left(\mathrm{F}_{\mathrm{HS}}\right)$. Open diamonds are for zero dipolar colloids; full diamonds are for 380D colloids; squares are for 490D colloids (colloids are type C, $\mathrm{D}$, and $\mathrm{E}$ in Table 1, respectively). Lines are guides to the eye.

Figure 5. Reduced total internal energy for a pair of interacting $+8 \mathrm{e}_{0}$ colloids as a function of reduced colloid-colloid separation. $\sigma_{C}$ is colloid diameter. Open diamonds are for zero dipolar (case C) colloids; full diamonds are for 380D colloids (case D); squares are for 490D colloids (case E). 
Figure 6. Simulated colloid-colloid reduced force as a function of reduced colloid-colloid separation for $+8 \mathrm{e}_{0}$ zero dipolar colloids (case $\mathrm{C}$ ) in electrolyte solution of various ionic strengths. $l_{B}$ is Bjerrum length, and $\sigma_{C}$ is colloid diameter. Fig. 6A shows the total colloid-colloid force $\left(\mathrm{F}_{\text {tot }}\right)$; Fig. 6B shows the electrostatic component of the total force $\left(\mathrm{F}_{\mathrm{el}}\right)$; and Fig. 6C shows the hard-sphere-collision component $\left(\mathrm{F}_{\mathrm{HS}}\right)$. Open symbols are for colloids in a $0.12 \mathrm{M}$ electrolyte solution; full symbols are for colloids in a $0.22 \mathrm{M}$ electrolyte solution. Diamonds are for colloids interacting in monovalent electrolyte solutions; squares are for colloids interacting in divalent electrolyte solutions. Lines are guides to the eye.

Figure 7. Simulated colloid-colloid reduced force as a function of reduced colloid-colloid separation for $+8 \mathrm{e}_{0}, 380 \mathrm{D}$ colloids (case D) in monovalent electrolyte solution. $l_{B}$ is Bjerrum length, and $\sigma_{C}$ is colloid diameter. Fig. 7A shows the total colloid-colloid force $\left(\mathrm{F}_{\text {tot }}\right)$; Fig. 7B shows the electrostatic component of the total force $\left(\mathrm{F}_{\mathrm{el}}\right)$, and Fig. $7 \mathrm{C}$ shows the hard-sphere-collision component $\left(\mathrm{F}_{\mathrm{HS}}\right)$. Open diamonds are for colloids in a $0.12 \mathrm{M}$ electrolyte solution, full diamonds are for colloids in a $0.22 \mathrm{M}$ electrolyte solution. Lines are guides to the eye.

Figure 8. Same as Fig. 7 for colloids interacting in divalent electrolyte solutions.

Figure 9. Reduced total internal energy for a pair of interacting $+8 \mathrm{e}_{0}$ colloids as a function of reduced colloid-colloid separation. $\sigma_{C}$ is colloid diameter. Figs. 9A and 9B are for zero dipolar and 380D colloids, respectively (cases C and D in table 1). Open symbols are for colloids interacting in $0.12 \mathrm{M}$ electrolyte solution, full symbols are for colloids interacting in $0.22 \mathrm{M}$ electrolyte solutions. Diamonds are for monovalent electrolyte solutions, squares are for divalent electrolyte solutions. Lines are guides to the eye.

Figure 10. Colloid-colloid potential of mean force from simulations (points) or from the DLVO theory (broken curves). Zero dipolar $+2 \mathrm{e}_{0}$ (case A) (open symbols) or $+8 \mathrm{e}_{0}$ (case C) (full symbols) colloids in $0.12 \mathrm{M}$ monovalent electrolyte solutions. $\sigma_{C}$ is colloid diameter.

Figure 11. Colloid-colloid potential of mean force from simulations (points), and from Eq.(3) (dashed curves), or from Eq.(10) (solid curves). Fig. $11 \mathrm{~A}$ is for $+8 \mathrm{e}_{0}, 380 \mathrm{D}$ 
colloids (case D in Table 1). Fig. 11B is for $+8 \mathrm{e}_{0}, 490 \mathrm{D}$ colloids (case E), all in monovalent $0.12 \mathrm{M}$ electrolyte solution. $\sigma_{C}$ is colloid diameter.

Figure 12. Colloid-colloid potential of mean force simulated (points) and calculated by theoretical models. Fig. 12A is for $+8 \mathrm{e}_{0}$ zero dipolar colloids (case $\mathrm{C}$ in Table 1); broken line is from DLVO theory; Fig. 12B is for $+8 \mathrm{e}_{0}, 380 \mathrm{D}$ colloids (case D); dashed line is from Eq.(3), and solid line is from Eq.(10). Colloids are simulated in divalent $0.12 \mathrm{M}$ electrolyte solution. $\sigma_{C}$ is colloid diameter. 

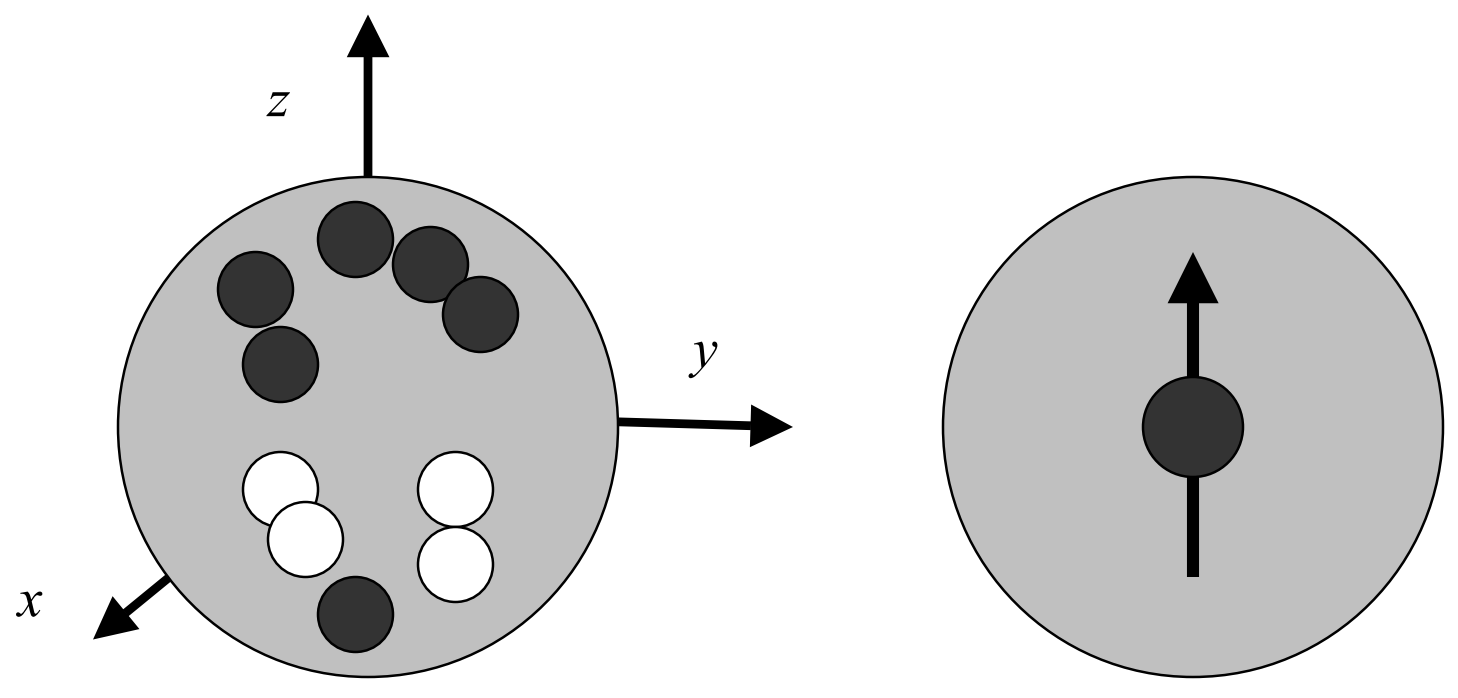

Figure 1. Striolo et al., 'Forces Between Aqueous ...' 
A)

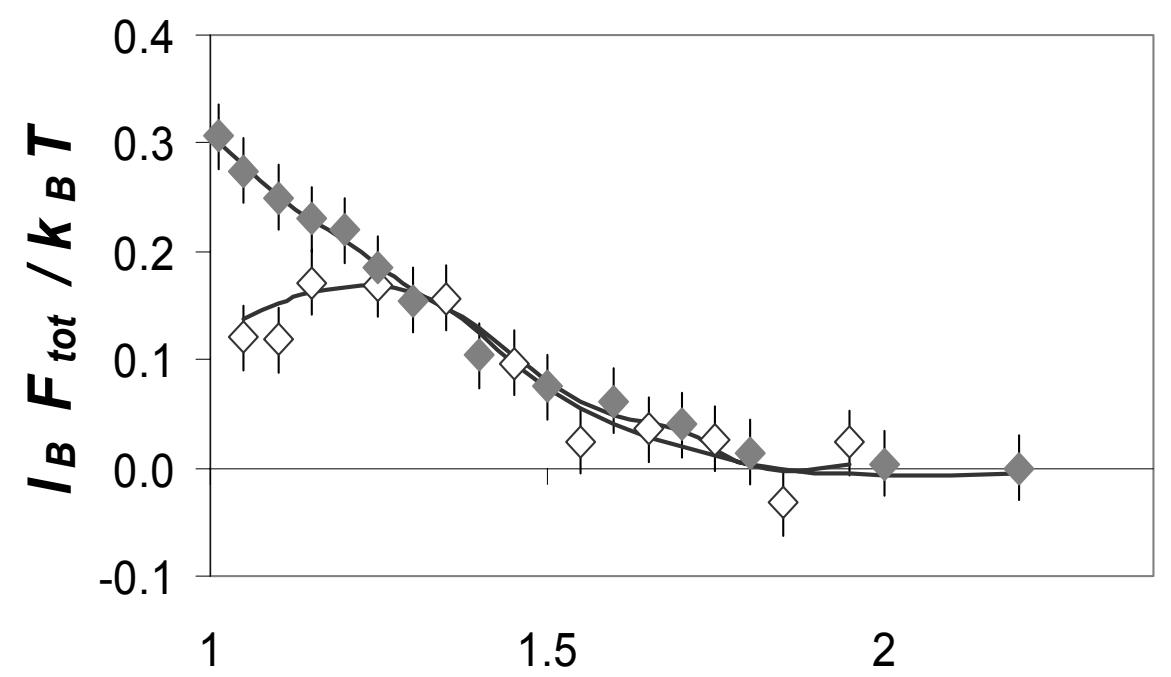

B)



C)

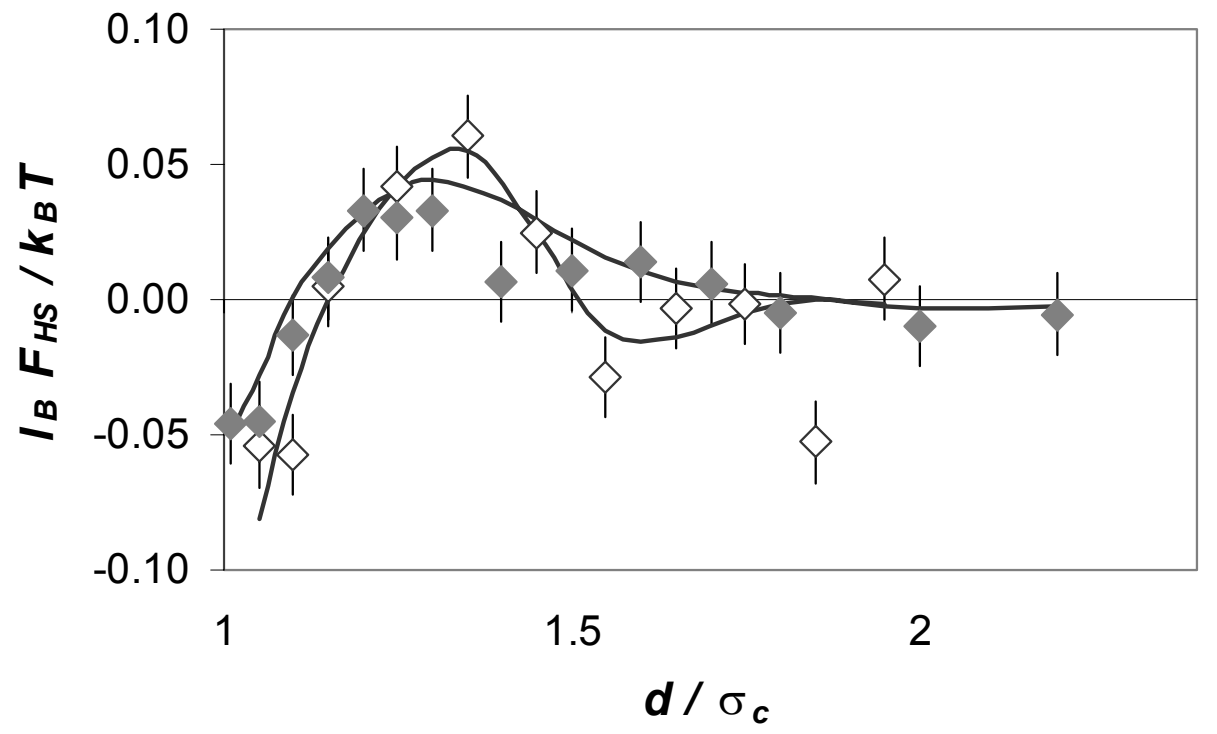

Figure 2. Striolo et al., 'Forces Between Aqueous ...' 


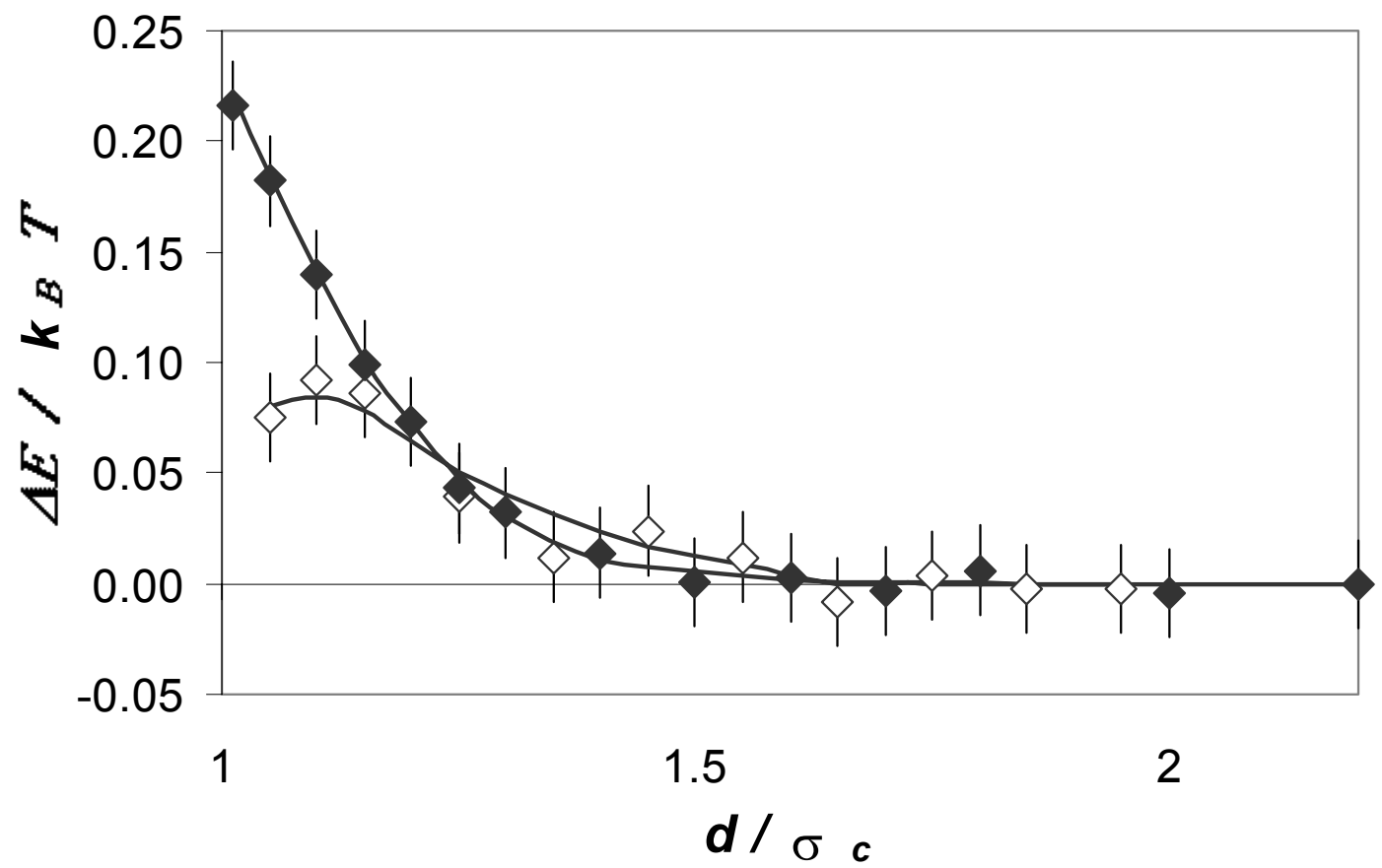

Figure 3. Striolo et al., 'Forces Between Aqueous ...' 
A)

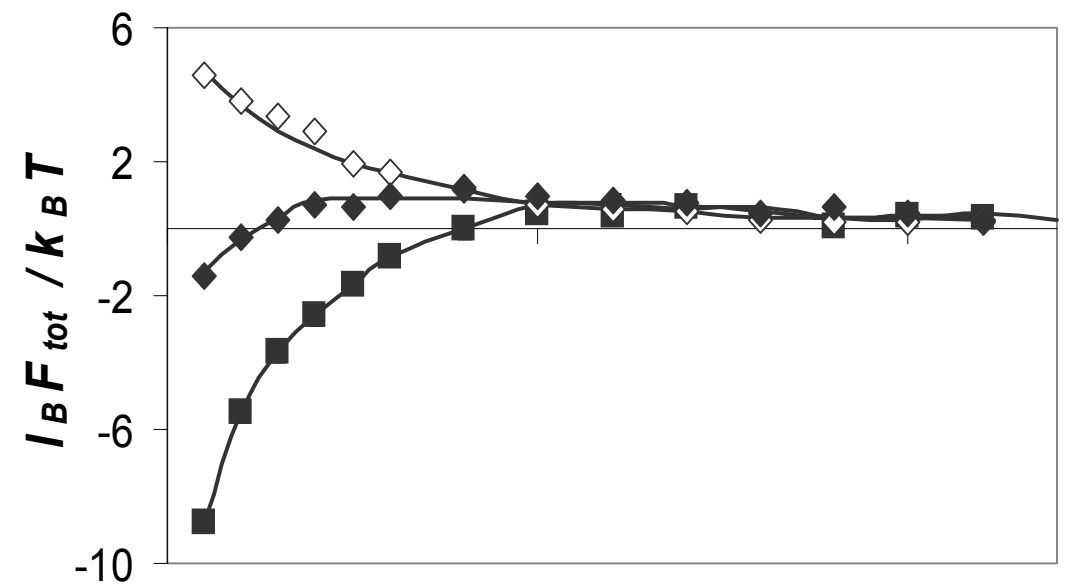

B)



C)

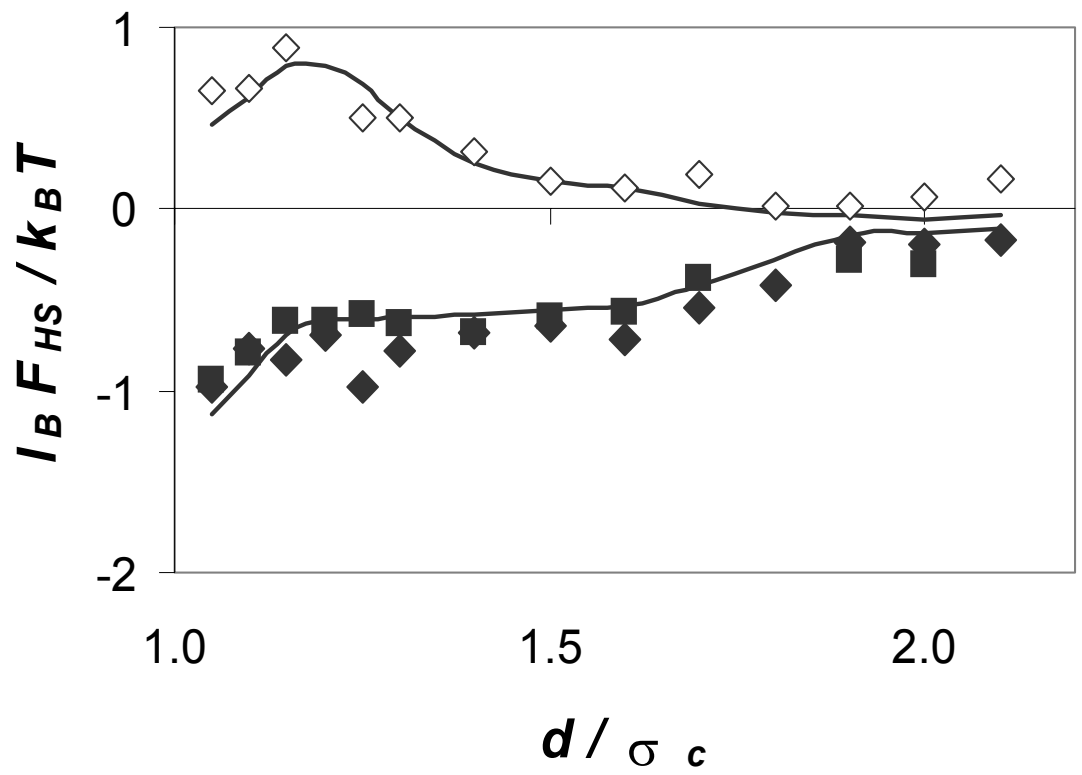

Figure 4. Striolo et al., 'Forces Between Aqueous ...' 


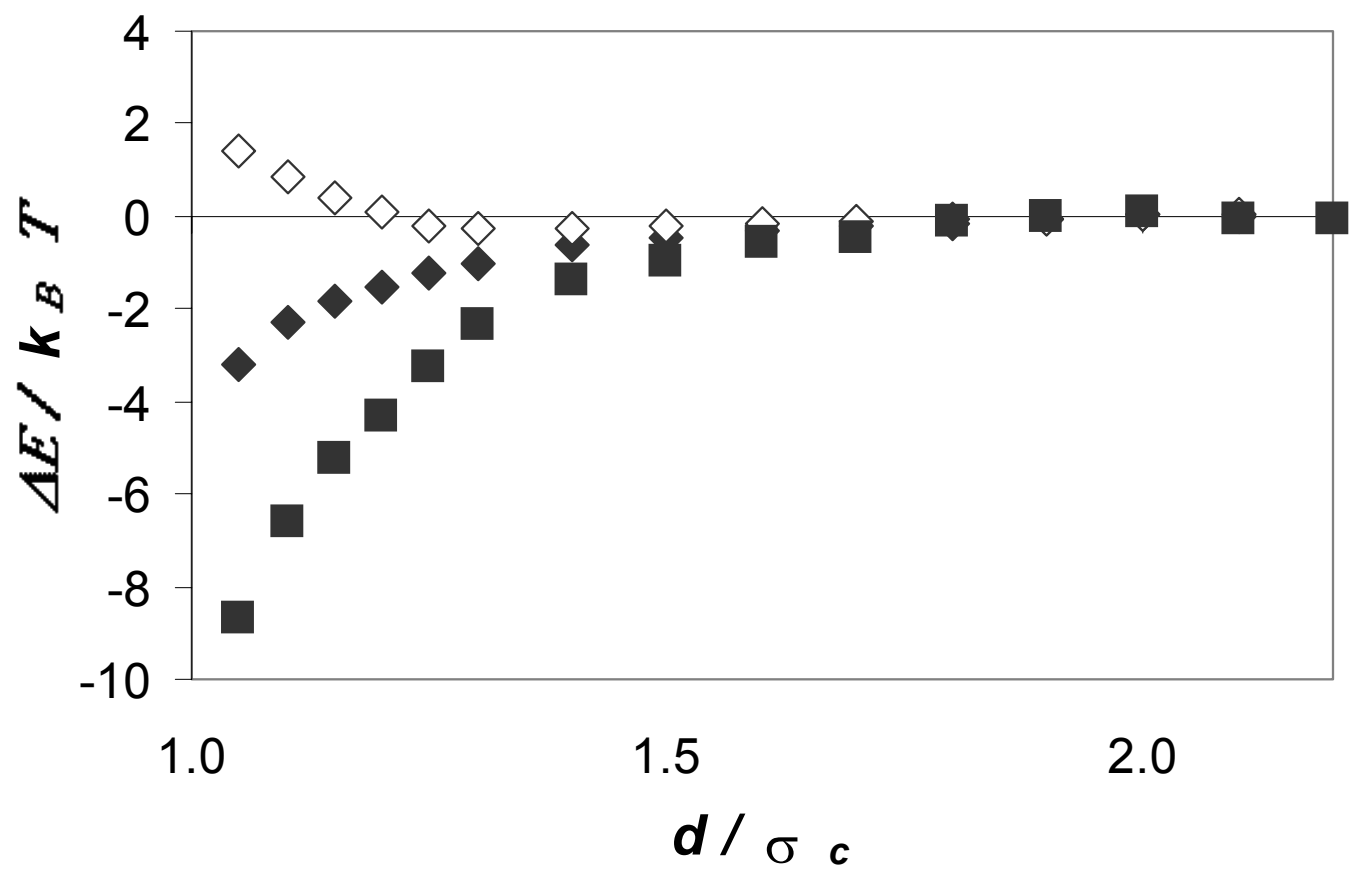

Figure 5. Striolo et al., 'Forces Between Aqueous ...' 
A)

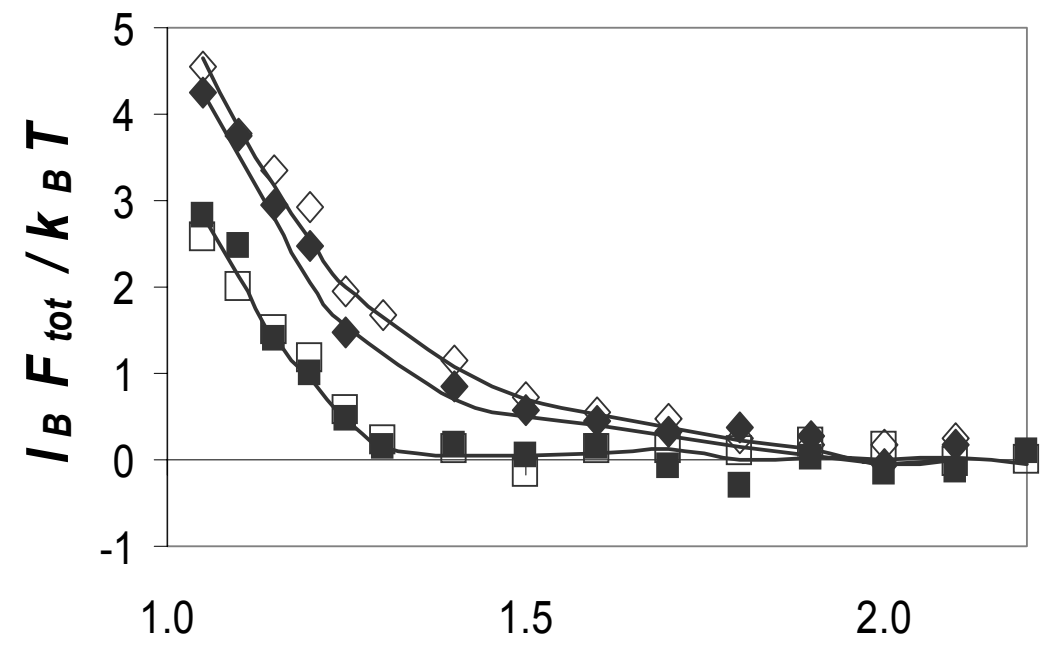

B)

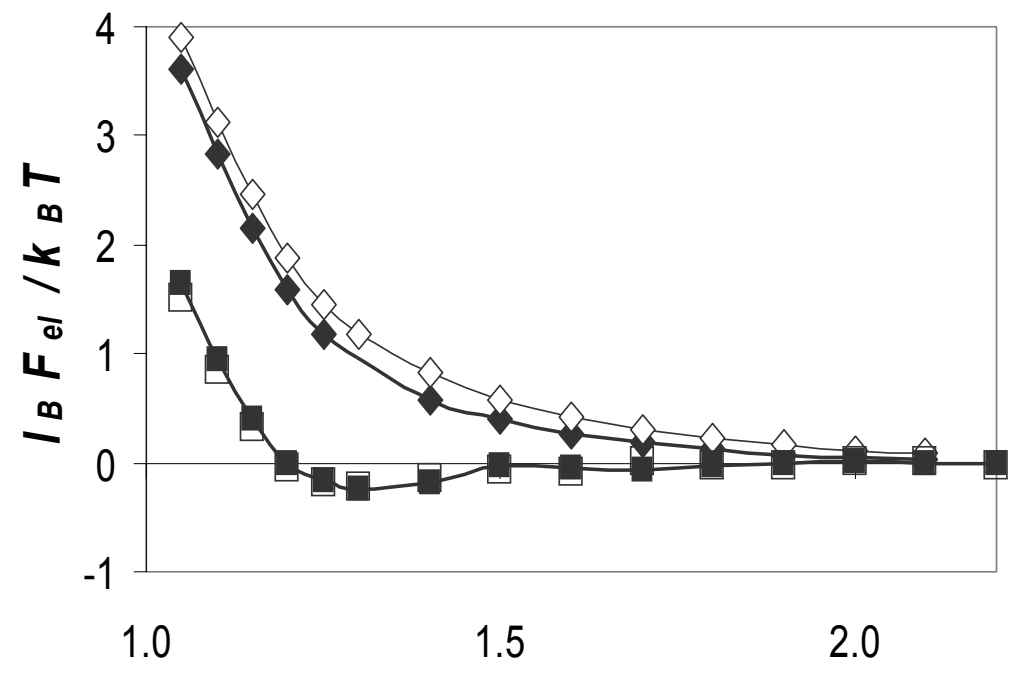

C)

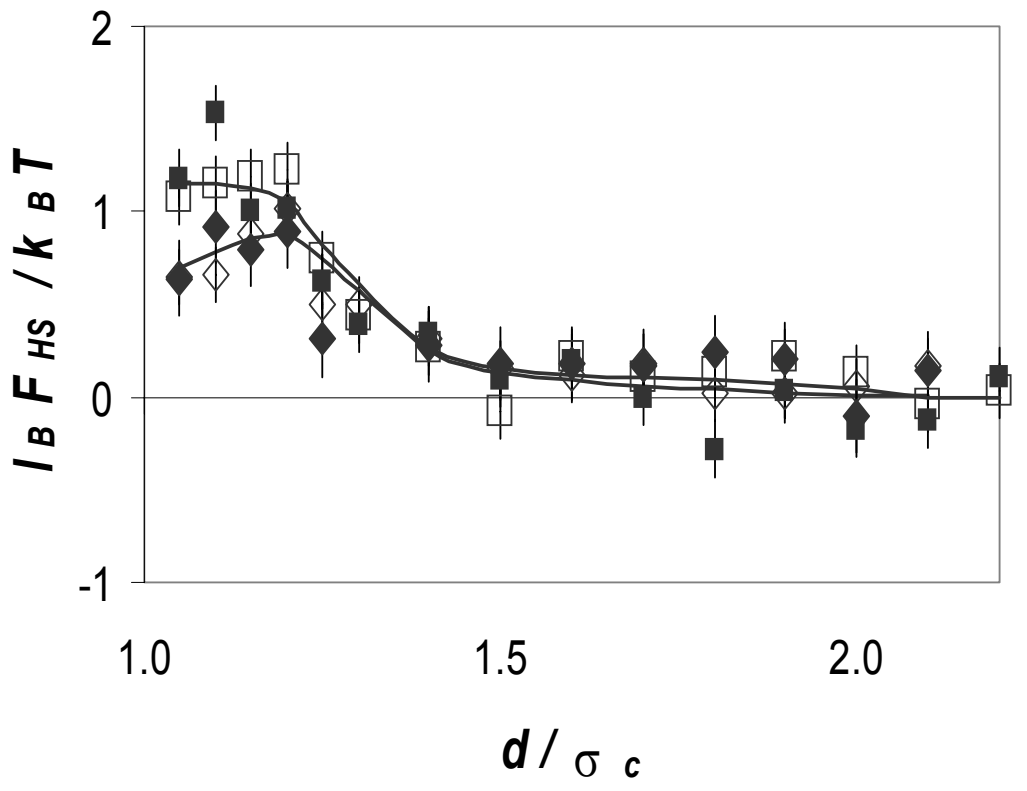

Figure 6. Striolo et al., 'Forces Between Aqueous ...' 
A)

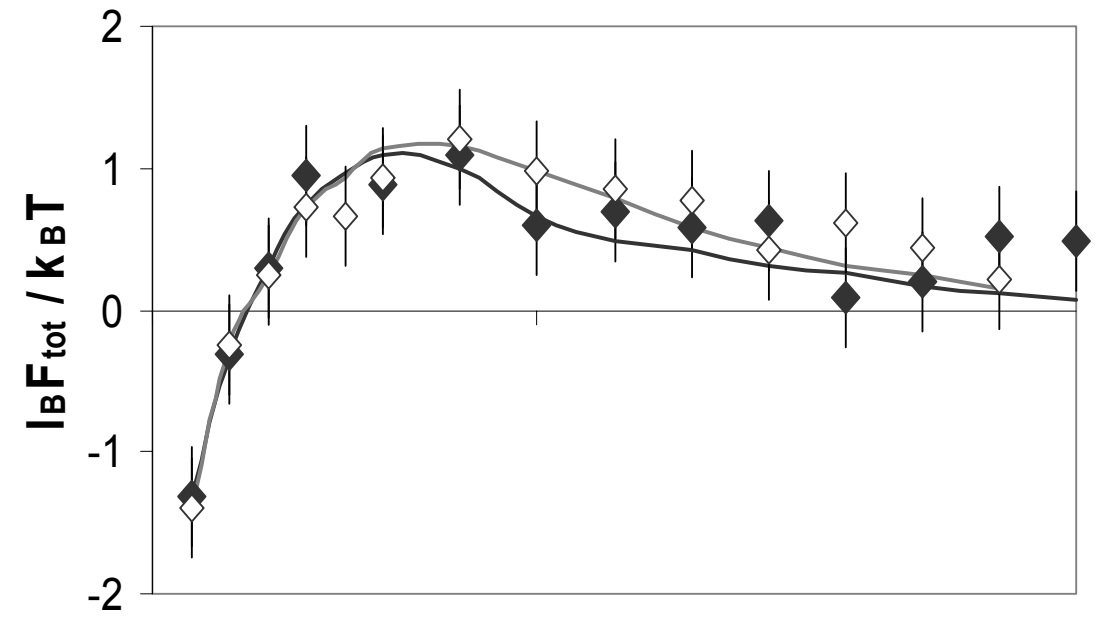

B)
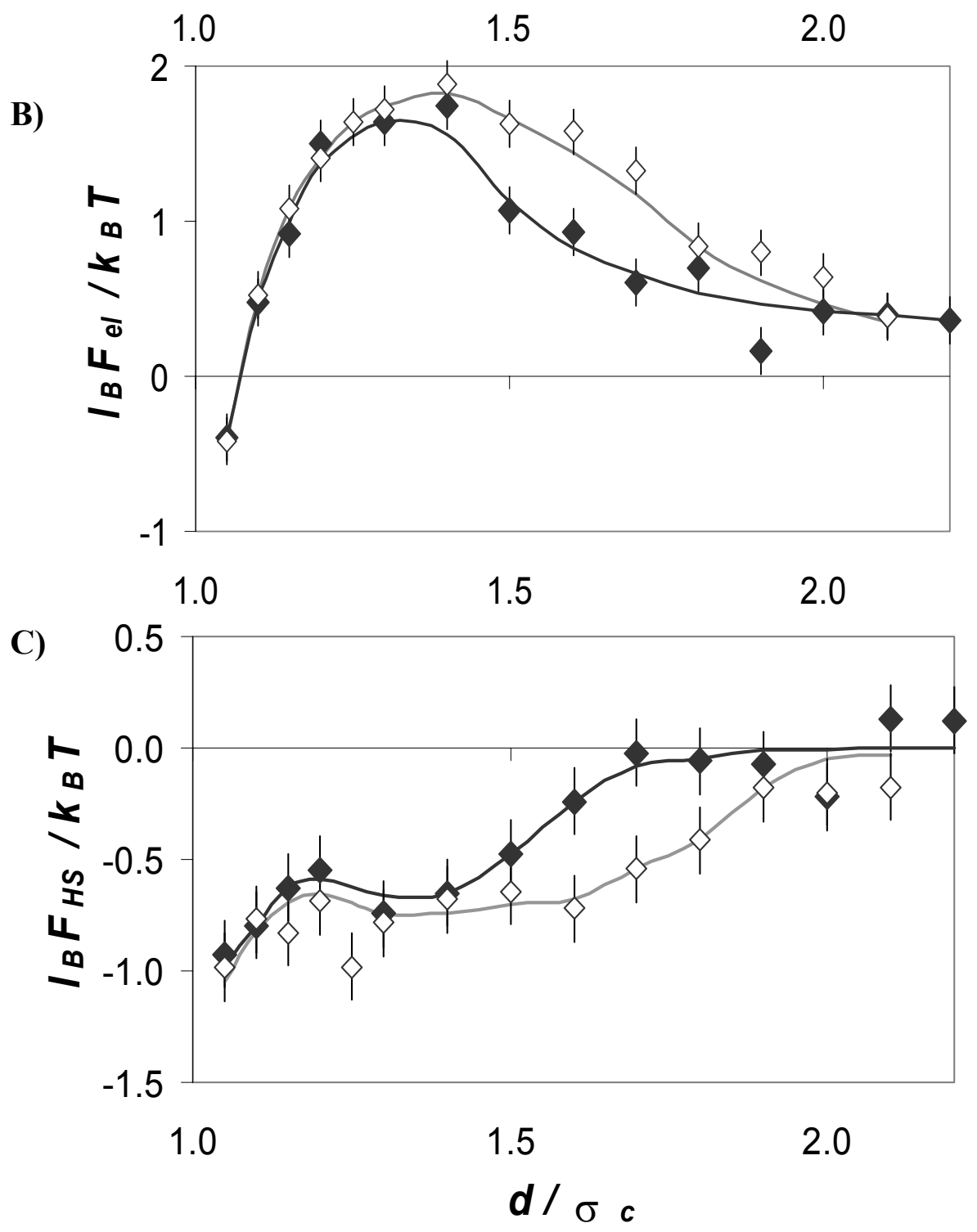

Figure 7. Striolo et al., 'Forces Between Aqueous ...' 
A)

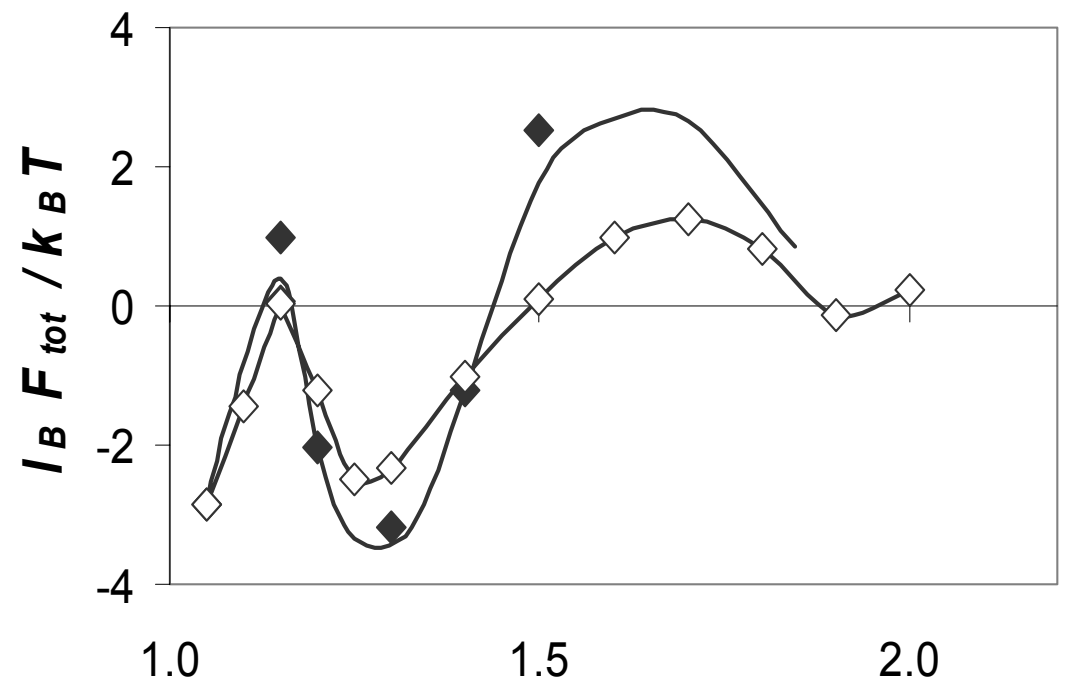

B)

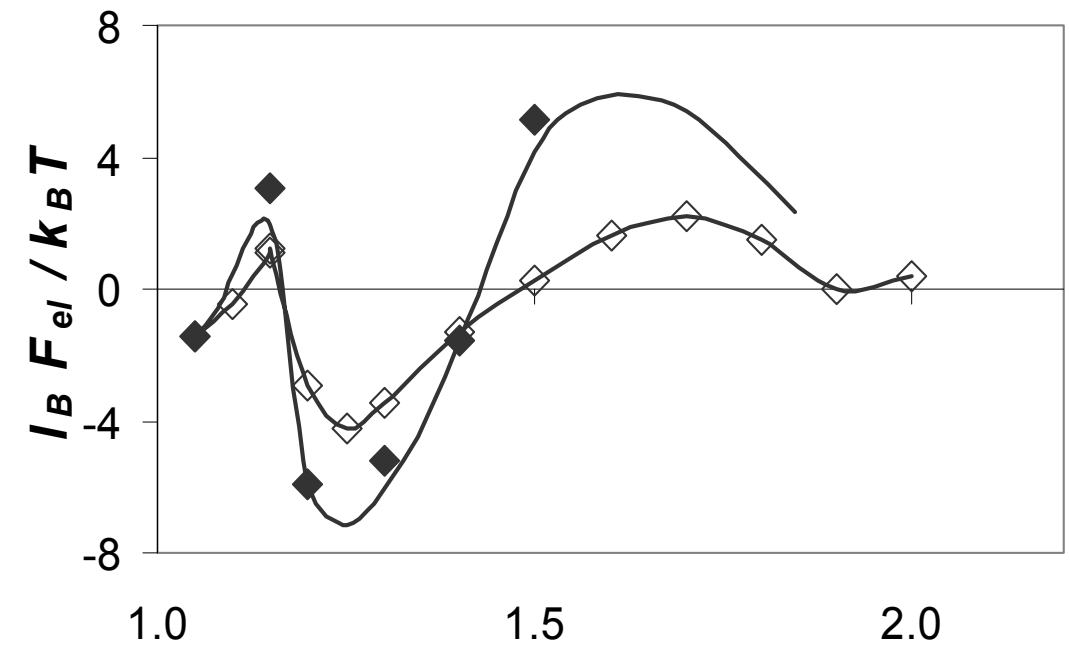

C)

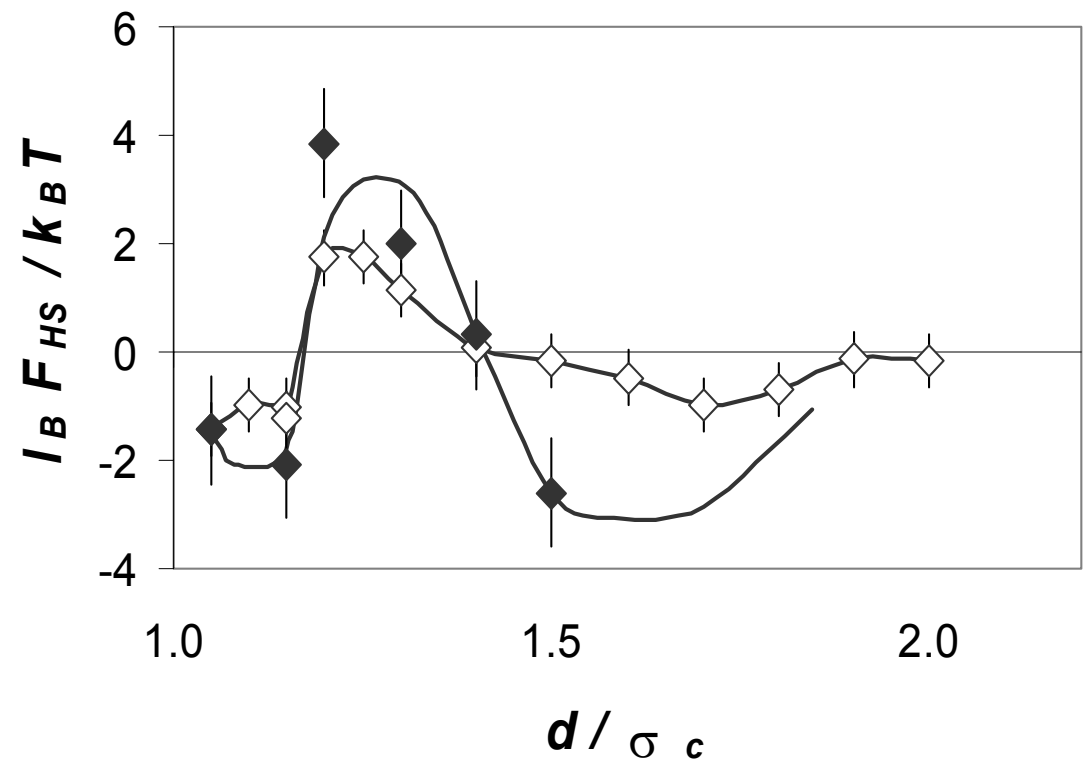

Figure 8. Striolo et al., 'Forces Between Aqueous ...' 

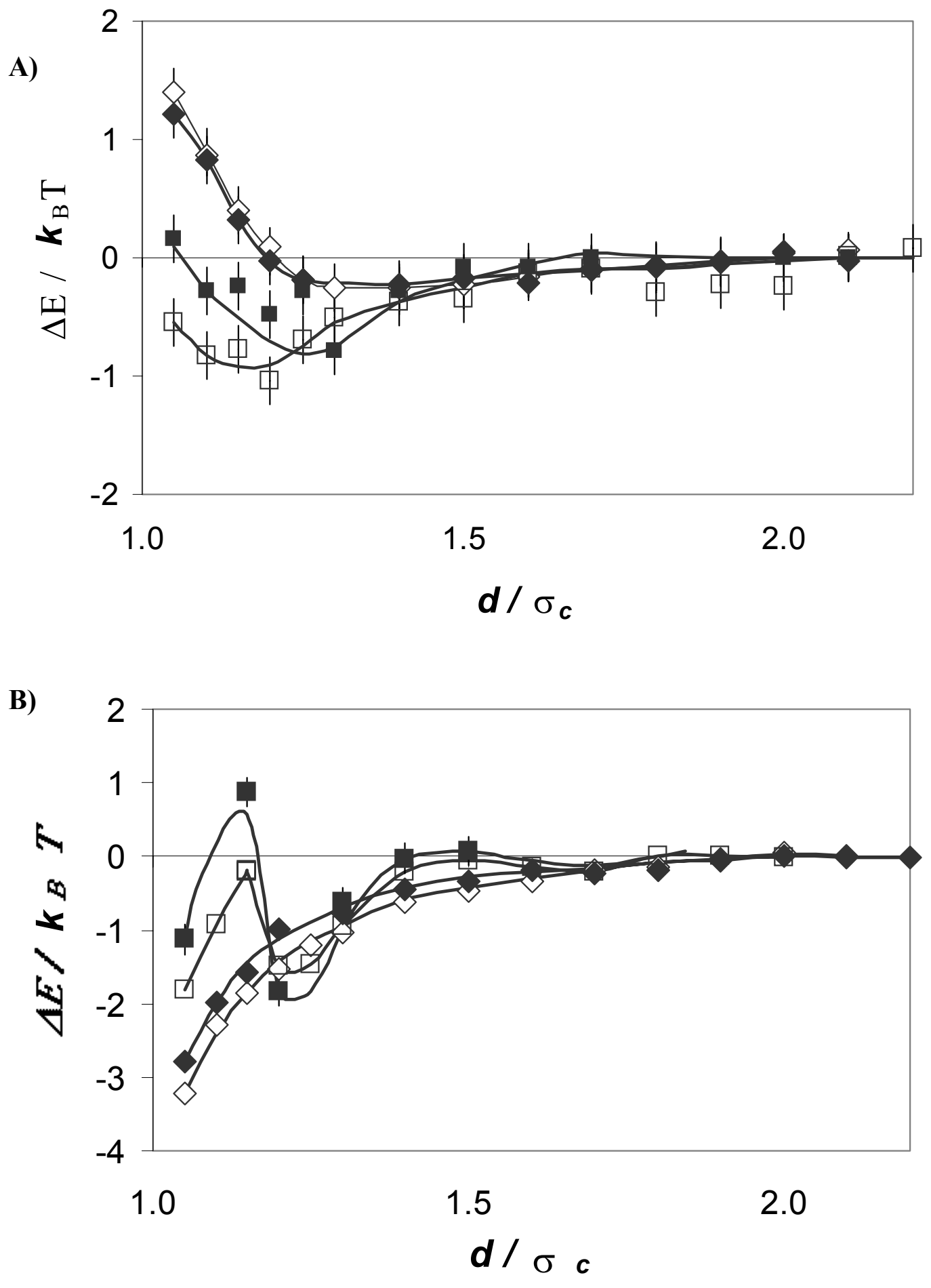

Figure 9. Striolo et al., 'Forces Between Aqueous ...' 


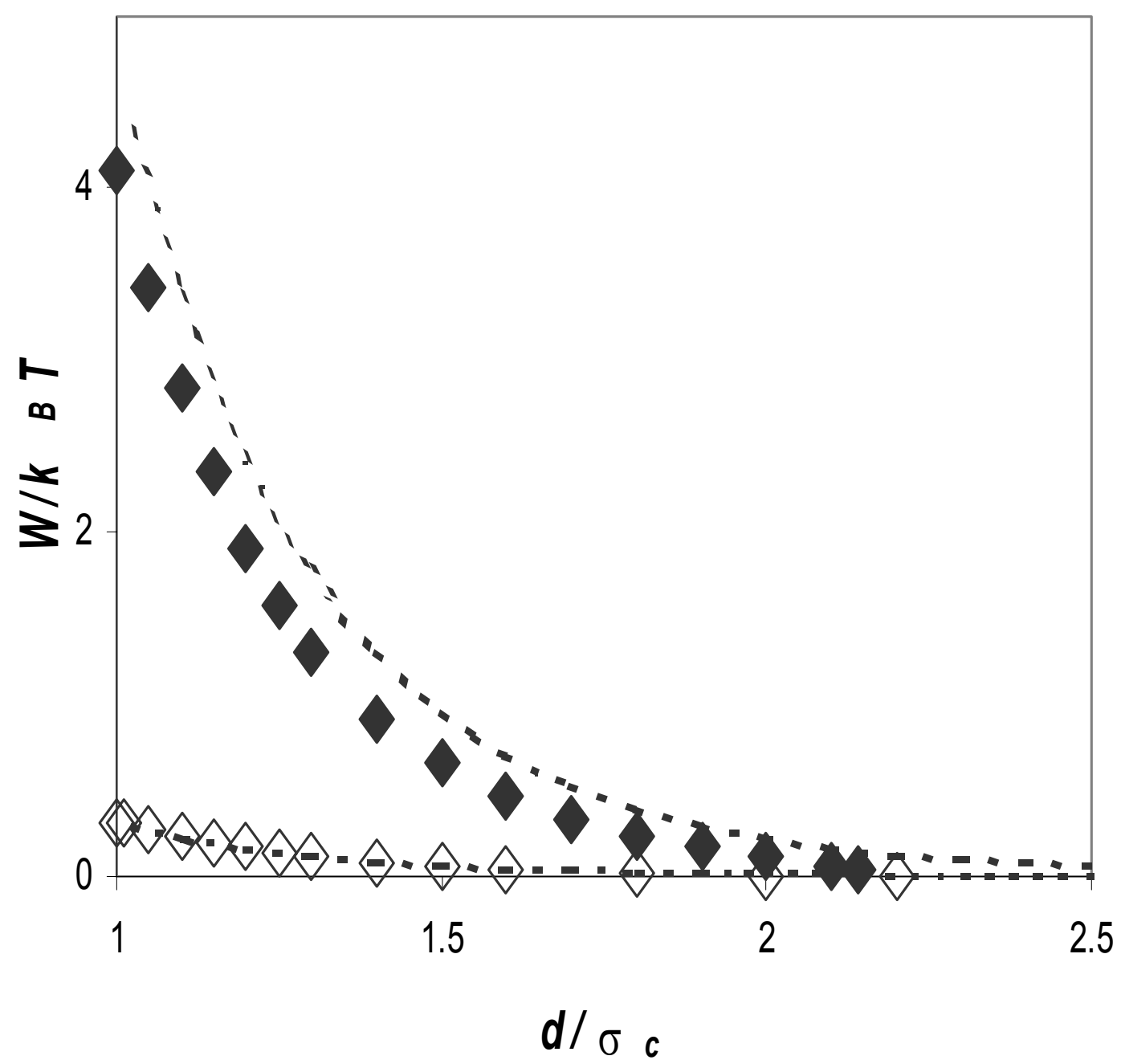

Figure 10. Striolo et al., 'Forces Between Aqueous ...' 
A)



B)

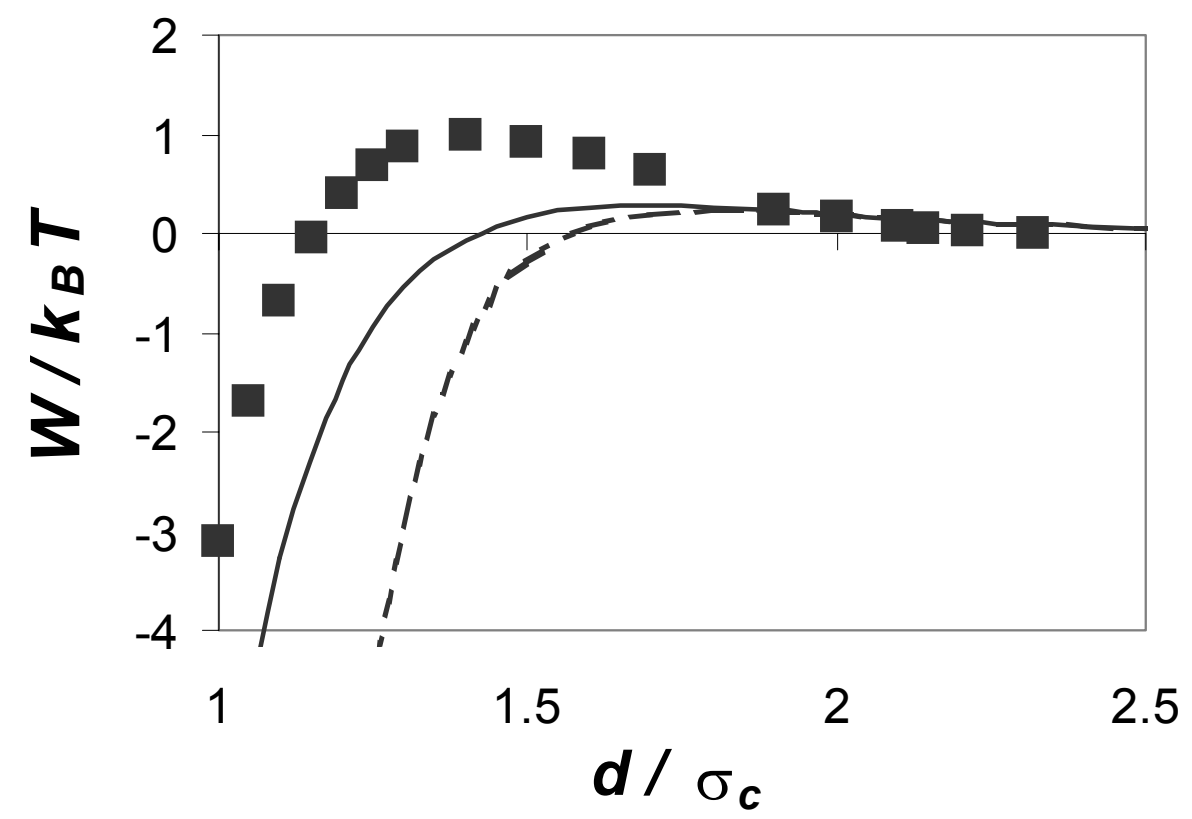

Figure 11. Striolo et al., 'Forces Between Aqueous ...' 
A)

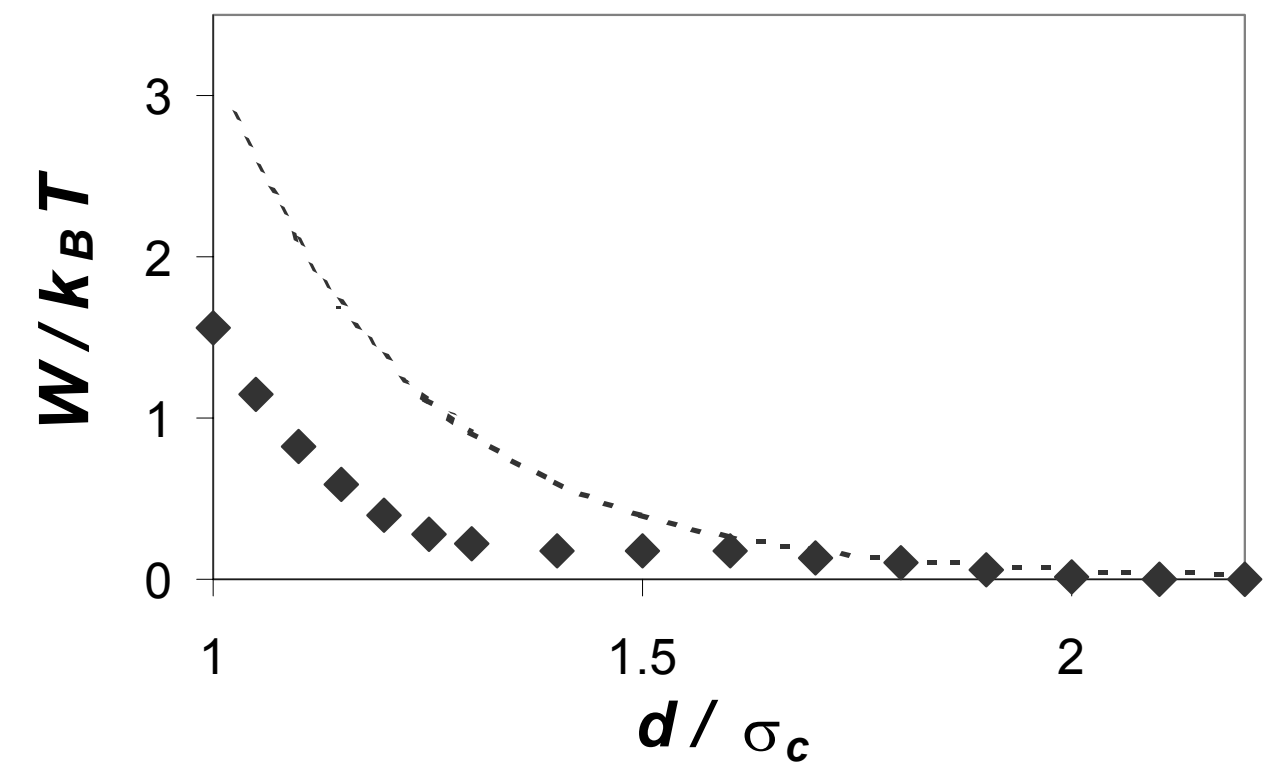

B)

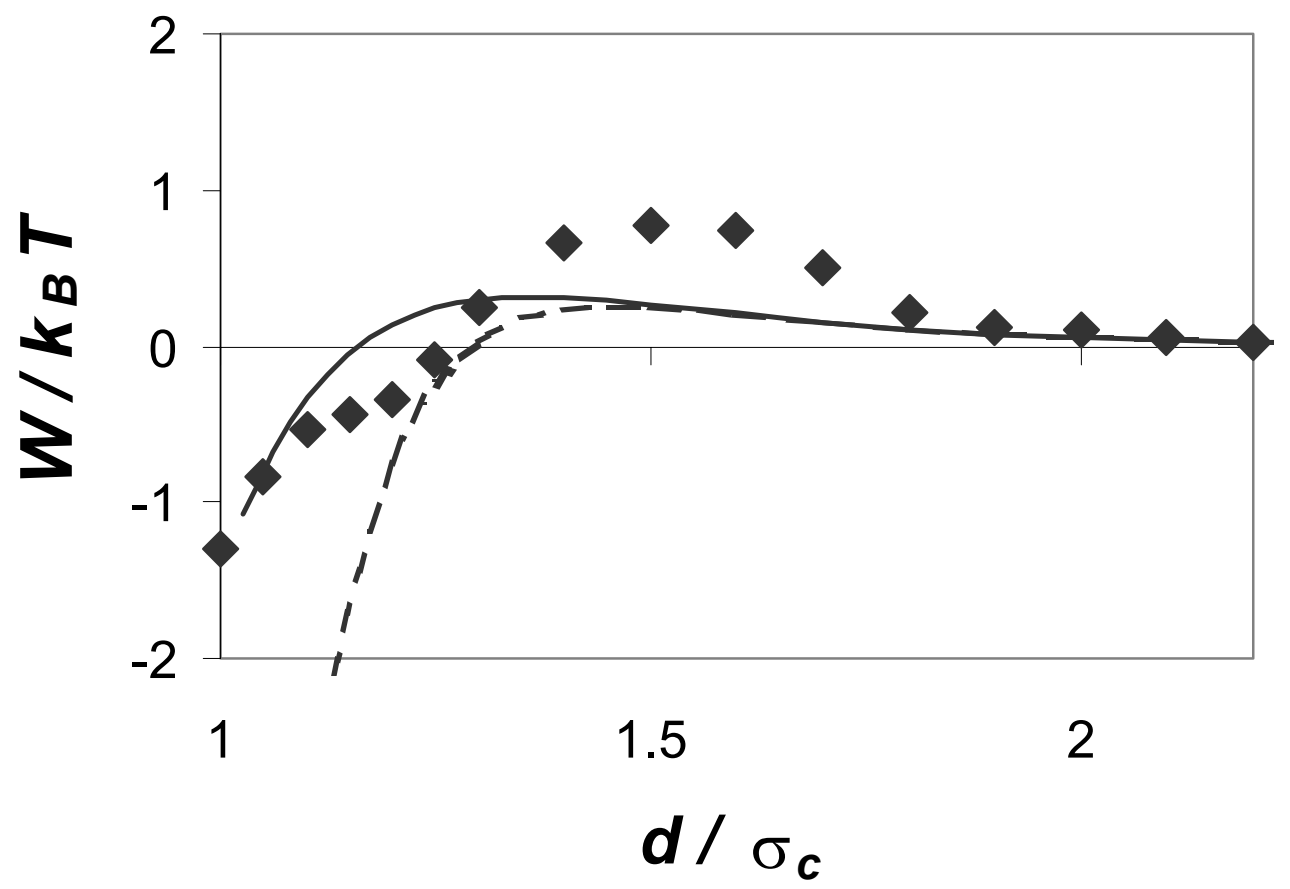

Figure 12. Striolo et al., 'Forces Between Aqueous ...' 